\title{
Prognostic value of microRNAs in gastric cancer: a meta-analysis
}

\author{
Yue Zhang ${ }^{1}$, Dong-Hui Guan'², Rong-Xiu $\mathrm{Bi}^{2}$, Jin $\mathrm{Xie}^{2}$, Chuan-Hua Yang ${ }^{3}$ and Yue- \\ Hua Jiang 4 \\ ${ }^{1}$ First Clinical Medical College, Shandong University of Traditional Chinese Medicine, Jinan 250355, Shandong, People's Republic \\ of China \\ ${ }^{2}$ Department of Orthopedics, Affiliated Hospital of Shandong University of Traditional Chinese Medicine, Jinan 250011, Shandong, \\ People's Republic of China \\ ${ }^{3}$ Department of Cardiology, Affiliated Hospital of Shandong University of Traditional Chinese Medicine, Jinan 250011, \\ Shandong, People's Republic of China \\ ${ }^{4}$ Central Laboratory, Affiliated Hospital of Shandong University of Traditional Chinese Medicine, Jinan 250011, Shandong, \\ People's Republic of China
}

Correspondence to: Yue-Hua Jiang, email: jiang_yuehua@hotmail.com

Keywords: microRNA, gastric cancer, prognosis, meta-analysis

Received: March 31, $2017 \quad$ Accepted: May 08, $2017 \quad$ Published: June 21, 2017

Copyright: Zhang et al. This is an open-access article distributed under the terms of the Creative Commons Attribution License 3.0 (CC BY 3.0), which permits unrestricted use, distribution, and reproduction in any medium, provided the original author and source are credited.

\section{ABSTRACT}

Background: Previous articles have reported that expression levels of microRNAs (miRNAs) are associated with survival time of patients with gastric cancer (GC). A systematic review and meta-analysis was performed to study the outcome of it.

Design: Meta-analysis.

Methods: English studies estimating expression levels of miRNAs with any of survival curves in GC were identified up till March 19, 2017 through performing online searches in PubMed, EMBASE, Web of Science and Cochrane Database of Systematic Reviews by two authors independently. The pooled hazard ratios (HR) with $95 \%$ confidence intervals (CI) were used to estimate the correlation between miRNA expression and overall survival (OS).

Results: Sixty-nine relevant articles about 26 miRNAs with 6148 patients were ultimately included. GC patients with high expression of miR-20b (HR=2.38, $95 \% C I=1.16-4.87), 21(H R=1.77,95 \% C I=1.01-3.08), 106 b \quad(H R=1.84,95 \% C I=1.15-$ $2.94), 196 \mathrm{a}(\mathrm{HR}=2.66,95 \% \mathrm{CI}=1.94-3.63), 196 \mathrm{~b}(\mathrm{HR}=1.67,95 \% \mathrm{CI}=1.38-2.02), 214$ $(H R=1.84,95 \% C I=1.27-2.67)$ or low expression of $m i R-125 a(H R=2.06,95 \% C I=1.26-$ $3.37), 137(\mathrm{HR}=3.21,95 \% \mathrm{CI}=1.68-6.13), 141(\mathrm{HR}=2.47,95 \% \mathrm{CI}=1.34-4.56), 145$ $(H R=1.62,95 \% C I=1.07-2.46), 146 a(H R=2.60,95 \% C I=1.63-4.13), 206(H R=2.85$, 95\%CI =1.73-4.70), $218(\mathrm{HR}=2.61,95 \% \mathrm{CI}=1.74-3.92), 451(\mathrm{HR}=1.73,95 \% \mathrm{CI}=1.19-$ 2.52), 486-5p $(H R=2.45,95 \% C I=1.65-3.65), 506(H R=2.07,95 \% C I=1.33-3.23)$ have significantly poor OS $(\mathrm{P}<0.05)$.

Conclusions: In summary, miR-20b, 21, 106b, 125a, 137, 141, 145, 146a, 196a, $196 b, 206,214,218,451,486-5 p$ and 506 demonstrate significantly prognostic value. Among them, miR-20b, 125a, 137, 141, 146a, 196a, 206, 218, 486-5p and 506 are strong biomarkers of prognosis in GC.

\section{INTRODUCTION}

Great quantities of previous articles have reported that expression levels of microRNAs (miRNAs) are associated with survival time of gastric cancer (GC) patients [1-167]. GC is still the fourth most common cancer all over the world and the second most universal cause of cancer death globally, although there has been a constant descent in morbidity and mortality in the past few decades $[168,169]$. The early clinical inspection of GC 
was under $15 \%$, and cases of advanced GC accounted for $85 \%$ [170]. At present, the primary treatment choices are surgical intervention, chemotherapy, immunogene therapy, and target therapy. The clinical result of GC mainly depends on the stage of tumor. Unfortunately, GC patients' median survival time is no more than 6-9 months [171]. It is unlimited proliferation of cancer cells and ability of intense invasive and metastasis that mainly causes high malignancy degree and poorer survival time. As a result, a novel diagnostic means and improved prognosis of GC might be created through identification of molecular aberrations, which can predict cancer progression and survival rate.

During the past decade, the associations between non-coding RNAs (ncRNAs) and GC have been widely researched. Generally speaking, ncRNAs have been classified as small ncRNAs, consisting of miRNAs and long non-coding RNAs (lncRNAs).

MiRNAs, a novel class of small (20-24 nucleotides [nt]) non-coding regulatory RNAs, play a significant role in multiple biological processes, such as cell division, differentiation, senescence and apoptosis [172, 173]. An increasing number of evidence shows that various miRNAs are unconventionally expressed in diverse types of human cancers, and a few miRNAs have been shown to be related with tumor formation, development, progression, and response to treatment by miRNA expression profiling [174].

Moreover, a series of studies have already demonstrated that lncRNAs also play crucial roles in GC progression. A previous investigation reported that, compared with non-tumor tissues, H19 was one of the

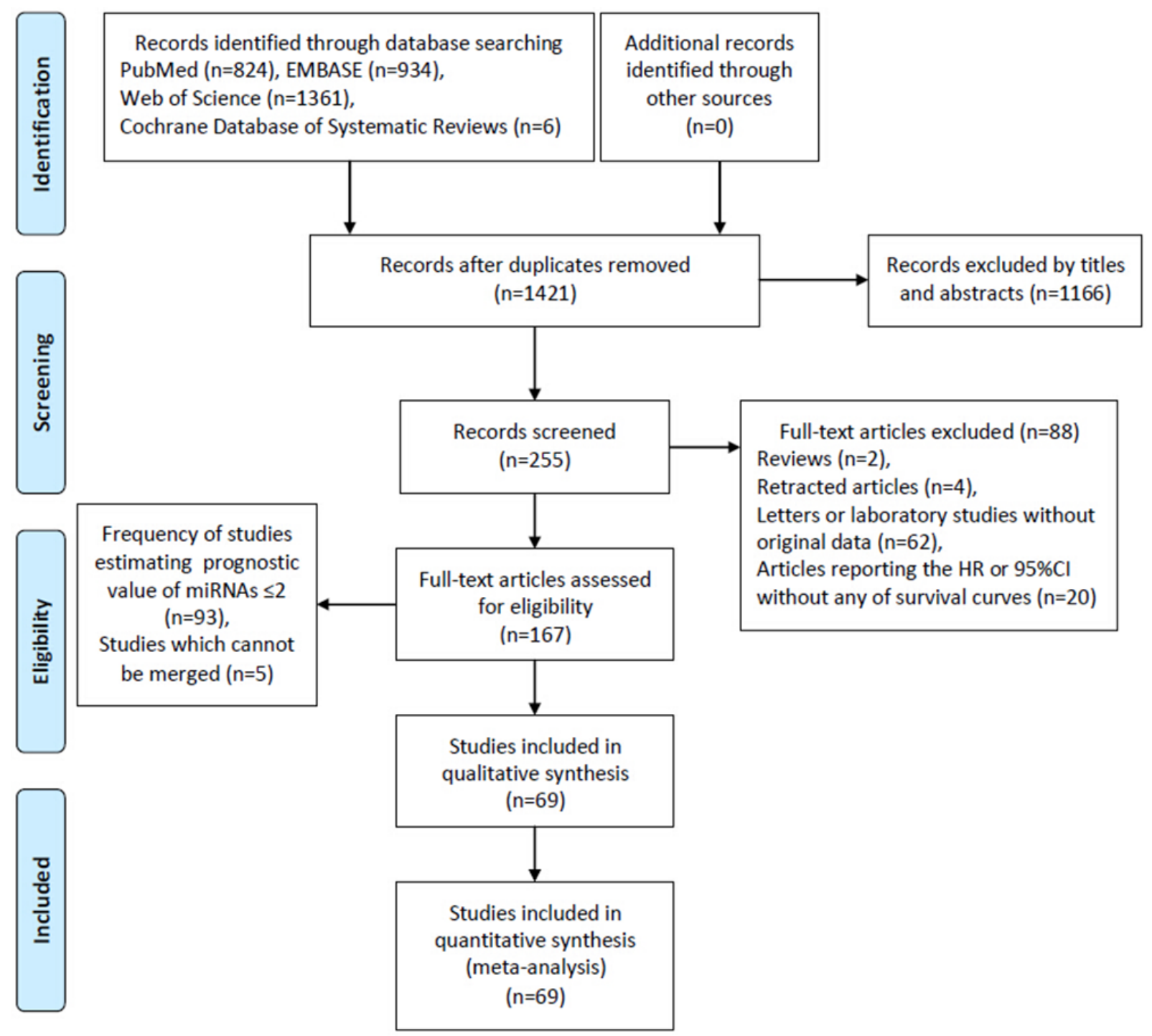

Figure 1: Flow diagram of literature search and selection. 
Table 1: Frequency of studies estimating prognostic value of miRNAs in gastric cancer

\begin{tabular}{|c|c|c|c|c|c|c|c|c|c|c|c|c|c|c|c|c|c|c|c|c|}
\hline miRNA & $\mathbf{N}$ & $\mathbf{R}$ & miRNA & $\mathbf{N}$ & $\mathbf{R}$ & miRNA & $\mathbf{N}$ & $\mathbf{R}$ & miRNA & $\mathbf{N}$ & $\mathbf{R}$ & miRNA & $\mathbf{N}$ & $\mathbf{R}$ & miRNA & $\mathbf{N}$ & $\mathbf{R}$ & miRNA & $\mathbf{N}$ & $\mathbf{R}$ \\
\hline let- $7 \mathrm{~g}$ & 1 & 1 & $27 \mathrm{~b}$ & 3 & $32-34$ & 126 & 2 & 54,55 & $148 \mathrm{a}$ & 1 & 78 & $200 \mathrm{a}$ & 1 & 3 & 328 & 1 & 126 & $485-5 p$ & 1 & 146 \\
\hline $10 \mathrm{~b}$ & 1 & 2 & $29 \mathrm{a}$ & 2 & 35,36 & 128 & 2 & 32,34 & $150-5 p$ & 1 & 77 & $200 \mathrm{~b}$ & 2 & 96,97 & 335 & 3 & $41,127,128$ & $486-5 p$ & 3 & $56,147,148$ \\
\hline $15 \mathrm{a}$ & 1 & 3 & $29 b$ & 1 & 36 & $129-5 p$ & 1 & 56 & 150 & 2 & 12,79 & $200 \mathrm{c}$ & 4 & $66,96,98,99$ & $337-3 p$ & 1 & 129 & 493 & 1 & 149 \\
\hline 16 & 2 & 3,4 & $29 \mathrm{c}$ & 1 & 36 & $130 \mathrm{a}$ & 1 & 57 & 153 & 1 & 80 & 203 & 2 & 100,101 & 340 & 1 & 130 & 494 & 1 & 150 \\
\hline $17-5 \mathrm{p}$ & 2 & 5,6 & 29 & 1 & 19 & 132 & 1 & 58 & $\begin{array}{c}181 \mathrm{a}- \\
5 \mathrm{p}\end{array}$ & 1 & 81 & 204 & 2 & 102,103 & $342-3 p$ & 1 & 79 & 500 & 1 & 151 \\
\hline $18 \mathrm{a}$ & 2 & 7,8 & 31 & 1 & 37 & $133 a-3 p$ & 1 & 56 & $181 \mathrm{~b}$ & 1 & 16 & 206 & 3 & $104-106$ & $361-5 p$ & 1 & 131 & $501-5 \mathrm{p}$ & 1 & 152 \\
\hline $19 \mathrm{a}$ & 1 & 9 & $34 \mathrm{a}$ & 5 & $3,38-41$ & 133 & 2 & 59,60 & $181 \mathrm{c}$ & 1 & 82 & 210 & 1 & 107 & 363 & 1 & 132 & 503 & 1 & 153 \\
\hline $19 b$ & 1 & 10 & $92 a$ & 2 & 11,42 & $135 \mathrm{a}$ & 1 & 61 & $182-5 p$ & 1 & 56 & 211 & 1 & 108 & 375 & 1 & 68 & 506 & 3 & $154-156$ \\
\hline $20 \mathrm{a}$ & 3 & $3,5,11$ & 93 & 2 & 43,44 & $135 b-5 p$ & 1 & 56 & $183-5 p$ & 1 & 56 & 212 & 1 & 109 & 377 & 1 & 133 & $508-5 \mathrm{p}$ & 2 & 33,157 \\
\hline $20 \mathrm{~b}$ & 3 & $3,12,13$ & 100 & 1 & 34 & $135 \mathrm{~b}$ & 1 & 61 & 183 & 2 & 83,84 & 214 & 4 & $1,34,110,111$ & 378 & 1 & 134 & $520 \mathrm{c}$ & 1 & 158 \\
\hline $21-5 p$ & 1 & 14 & 101 & 2 & 32,34 & 137 & 3 & $62-64$ & 185 & 2 & 3,85 & 215 & 1 & 87 & 381 & 1 & 135 & $520 \mathrm{~d}-3 \mathrm{p}$ & 1 & 159 \\
\hline 21 & 7 & $\begin{array}{c}3,6,15- \\
19\end{array}$ & 103 & 1 & 3 & 141 & 3 & $65-67$ & 187 & 1 & 86 & 217 & 2 & 112,113 & 421 & 2 & 136,137 & 558 & 1 & 160 \\
\hline 22 & 2 & 20,21 & $106 \mathrm{a}$ & 2 & 3,6 & $142-5 p$ & 1 & 68 & 192 & 3 & $48,79,87$ & 218 & 3 & $114-116$ & 425 & 1 & 3 & $590-5 p$ & 1 & 161 \\
\hline $23 b-3 p$ & 1 & 22 & $106 \mathrm{~b}$ & 3 & $3,6,45$ & 143 & 3 & $3,69,70$ & $193 b$ & 1 & 88 & 221 & 2 & 117,118 & 429 & 1 & 138 & 630 & 1 & 162 \\
\hline $23 b$ & 1 & 23 & 107 & 3 & $3,46,47$ & $144-5 p$ & 1 & 56 & 194 & 1 & 89 & 222 & 2 & 118,119 & 433 & 1 & 1 & 873 & 1 & 163 \\
\hline 24 & 1 & 24 & 122 & 1 & 48 & 144 & 1 & 71 & $196 a$ & 4 & $\begin{array}{c}88,90- \\
92\end{array}$ & 223 & 2 & 120,121 & 448 & 1 & 139 & 939 & 1 & 164 \\
\hline 25 & 2 & 25,26 & $125 a-3 p$ & 1 & 49 & $145-5 p$ & 2 & 56,72 & $\begin{array}{c}196 \mathrm{~b}- \\
5 \mathrm{p}\end{array}$ & 1 & 56 & 224 & 2 & 79,122 & $449 \mathrm{c}$ & 1 & 140 & 940 & 1 & 165 \\
\hline $26 \mathrm{a}$ & 2 & 27,28 & $125 a-5 p$ & 1 & 50 & 145 & 2 & 34,73 & $196 \mathrm{~b}$ & 3 & $91-93$ & 300 & 1 & 123 & 451 & 4 & $4,141-143$ & $1207-5 p$ & 1 & 166 \\
\hline $26 \mathrm{~b}$ & 1 & 29 & $125 \mathrm{a}$ & 1 & 51 & $146 a$ & 3 & $74-76$ & 198 & 1 & 94 & $301 \mathrm{a}$ & 1 & 124 & 452 & 1 & 144 & $1225-5 p$ & 1 & 167 \\
\hline $27 \mathrm{a}$ & 2 & 30,31 & $125 \mathrm{~b}$ & 2 & 52,53 & $146 b-5 p$ & 1 & 77 & $199 \mathrm{a}$ & 1 & 95 & 326 & 1 & 125 & $455-5 p$ & 1 & 145 & 1266 & 1 & 166 \\
\hline
\end{tabular}

Highlighted studies were included in the present meta-analysis; N: Number of studies estimating prognostic value; R: Reference.

most elevated lncRNAs with a $~ 8.91$-fold change in human primary GC [175]. In addition, Li et al. [176] recognized certain potential IncRNAs that abnormally expressed between GC and normal tissues by screening a cohort of 74 GC patients as well, among which, H19 was chosen as a result of a significant overexpression. Furthermore, expression levels of the lncRNAs H19, ANRIL, GHET1, HOTAIR, GAS5, LET, GAPLINC and FENDRR are also significantly associated with the 5-year survival rate of GC patients [176-183].

In GC research area, quite a number of investigations have demonstrated that miRNAs are associated with survival time of patients [1-167]. However, the number of patients during the articles mentioned above is generally not big enough. Therefore, a systematic review and meta-analysis was performed for the sake of better understanding accurate prognostic value between expression levels of numerous miRNAs and HR of GC patients.

\section{RESULTS}

\section{Study selection}

A flow diagram with details of the study selection process was presented in Figure 1.

\section{Study frequency}

Frequency of studies estimating prognostic value of miRNAs in GC were shown in Table 1 (highlighted studies were included in the present meta-analysis), including miRNA name, number of studies estimating prognostic value, and reference.

\section{Study characteristics}

Characteristics of articles with Kaplan-Meier survival curves in GC were comprehensively detailed in Table 2, including miRNA name, names of the first authors, publication year, reference number, country, study design, detected sample, number of patients, stage, cut-off value, main miRNA method, maximum months of followup, survival analysis and HR of low or high expression on the basis of relevant survival analysis with $95 \% \mathrm{CI}$. If the data were not provided visually and only as KaplanMeier survival curves, the data were extracted from the graphical survival plots, and estimations of the HR with $95 \%$ CI were then performed using a previously described method [184] with the software Engauge Digitizer version 4.1. Furthermore, if both the univariate and multivariate results were reported, then only the latter was selected, since these results were adjusted for confounding factors. 
Table 2: Characteristics of articles with Kaplan-Meier survival curves in gastric cancer

\begin{tabular}{|c|c|c|c|c|c|c|c|c|c|c|c|c|c|}
\hline miRNA & Study & Country & $\begin{array}{l}\text { Study } \\
\text { design }\end{array}$ & Sample & Number & Stage & Cut-off & Method & $\begin{array}{l}\text { Follow-up } \\
\text { (month) }\end{array}$ & Result & $\begin{array}{c}\text { HR } \\
(\mathrm{L} / \mathrm{H})\end{array}$ & $\begin{array}{c}\text { HR } \\
(\mathrm{H} / \mathrm{L})\end{array}$ & $95 \%$ CI \\
\hline $20 \mathrm{a}$ & Osawa S, 2011 [3] & Japan & $\mathrm{R}$ & FFPE & 37 & II-III & $70 \%$ & qRT-PCR & 60 & $\mathrm{OS}^{\mathrm{u}}$ & & 1.93 & $0.48-7.87$ \\
\hline $20 \mathrm{a}$ & Wang M, 2012 [5] & China & $\mathrm{R}$ & Plasma & 65 & I-IV & 0.26 & RT-qPCR & 36 & $\mathrm{OS}^{\mathrm{m}}$ & & 1.58 & $1.10-2.25$ \\
\hline $20 \mathrm{a}$ & Wu Q, 2013 [11] & China & $\mathrm{R}$ & FFPE & 97 & None & Median & qRT-PCR & 66 & $\mathrm{OS}^{\mathrm{m}}$ & & 1.01 & $1.00-1.02$ \\
\hline $20 \mathrm{~b}$ & Katada T, 2009 [12] & Japan & $\mathrm{R}$ & Frozen & 42 & None & None & qRT-PCR & 60 & $\mathrm{OS}^{\mathrm{m}}$ & & 2.01 & $0.59-6.85$ \\
\hline $20 \mathrm{~b}$ & Osawa S, 2011 [3] & Japan & $\mathrm{R}$ & FFPE & 34 & II-III & $70 \%$ & qRT-PCR & 60 & $\mathrm{OS}^{\mathrm{u}}$ & & 1.21 & $0.20-7.23$ \\
\hline $20 \mathrm{~b}$ & Xue TM, 2015 [13] & China & $\mathrm{R}$ & Tissue & 102 & I-IV & Median & RT-qPCR & 75 & $\mathrm{OS}^{\mathrm{m}}$ & & 3.32 & $1.20-9.14$ \\
\hline $21-5 p$ & Park SK, 2016 [14] & Korea & $\mathrm{R}$ & FFPE & 50 & III & ROC & qRT-PCR & 168 & $\mathrm{RFS}^{\mathrm{u}}$ & & 2.05 & $1.26-3.34$ \\
\hline 21 & Jiang J, 2011 [15] & China & $\mathrm{R}$ & FFPE & 55 & III-IV & None & qRT-PCR & 17 & $\mathrm{OS}^{\mathrm{u}}$ & & 5.88 & $2.22-16.67$ \\
\hline 21 & Osawa S, 2011 [3] & Japan & $\mathrm{R}$ & FFPE & 33 & II-III & $70 \%$ & qRT-PCR & 60 & $\mathrm{OS}^{\mathrm{u}}$ & & 2.58 & $0.34-19.79$ \\
\hline 21 & $\mathrm{Xu} \mathrm{Y,} 2012$ [16] & China & $\mathrm{R}$ & Frozen & 86 & I-IV & 5.12 & qRT-PCR & 36 & $\mathrm{OS}^{u}$ & & 1.15 & $0.59-2.25$ \\
\hline 21 & Hirata K, 2013 [17] & Japan & $\mathrm{P}$ & Tissue & 61 & None & 3.58 & $\mathrm{IHC}$ & 42 & $\mathrm{RFS}^{u}$ & & 0.82 & $0.27-2.43$ \\
\hline 21 & Komatsu S, 2013 [6] & Japan & $\mathrm{R}$ & Plasma & 69 & I-IV & 0.03 & qRT-PCR & 40 & $\mathrm{CSS}^{\mathrm{m}}$ & & 13.39 & $1.72-104.42$ \\
\hline 21 & Song J, 2013 [18] & China & $\mathrm{R}$ & Serum & 103 & I-IV & 0.64 & qRT-PCR & 54 & $\mathrm{OS}^{\mathrm{u}}$ & & 0.99 & $0.48-2.07$ \\
\hline 21 & Wang D, 2015 [19] & China & $\mathrm{R}$ & Tissue & 50 & I-IV & ROC & qRT-PCR & 12 & $\mathrm{OS}^{\mathrm{u}}$ & & 1.89 & $1.17-3.07$ \\
\hline $27 b$ & Liu HT, 2015 [32] & China & $\mathrm{R}$ & FFPE & 103 & I-IV & None & qRT-PCR & 66 & $\mathrm{OS}^{\mathrm{u}}$ & 0.80 & & $0.46-1.41$ \\
\hline $27 b$ & Shang Y, 2016 [33] & China & $\mathrm{R}$ & Tissue & 114 & I-IV & None & ISH & 84 & $\mathrm{OS}^{\mathrm{u}}$ & 1.61 & & $0.92-2.80$ \\
\hline $27 \mathrm{~b}$ & Liu HT, 2017 [34] & China & $\mathrm{R}$ & FFPE & 102 & I-IV & Median & RT-qPCR & 67 & $\mathrm{OS}^{\mathrm{m}}$ & 1.33 & & $0.60-2.98$ \\
\hline $34 \mathrm{a}$ & Osawa S, 2011 [3] & Japan & $\mathrm{R}$ & FFPE & 37 & II-III & $70 \%$ & qRT-PCR & 60 & $\mathrm{OS}^{\mathrm{u}}$ & 0.20 & & $0.06-0.68$ \\
\hline $34 \mathrm{a}$ & Hui WT, 2015 [38] & China & $\mathrm{R}$ & Frozen & 76 & I-III & Mean & qRT-PCR & $>60$ & $\mathrm{OS}^{\mathrm{m}}$ & 2.33 & & $1.10-4.93$ \\
\hline $34 \mathrm{a}$ & Wei B, 2015 [39] & TCGA & $\mathrm{R}$ & Tissue & 157 & I-IV & X-tile & Downloaded & $>100$ & $\mathrm{OS}^{\mathrm{u}}$ & 2.31 & & $0.13-40.12$ \\
\hline $34 \mathrm{a}$ & Zhang H, 2015 [40] & China & $\mathrm{R}$ & Frozen & 137 & I-IV & 2.44 & qRT-PCR & 68 & $\mathrm{OS}^{\mathrm{m}}$ & 1.33 & & $1.14-1.61$ \\
\hline $34 \mathrm{a}$ & Yang B, 2016 [41] & China & $\mathrm{R}$ & Tissue & 50 & I-IV & Median & qRT-PCR & 60 & $\mathrm{OS}^{\mathrm{u}}$ & 3.05 & & $0.60-15.50$ \\
\hline $106 b$ & Osawa S, 2011 [3] & Japan & $\mathrm{R}$ & FFPE & 37 & II-III & $70 \%$ & qRT-PCR & 60 & $\mathrm{OS}^{\mathrm{u}}$ & & 2.70 & $0.43-17.06$ \\
\hline $106 b$ & Komatsu S, 2013 [6] & Japan & $\mathrm{R}$ & Plasma & 69 & I-IV & 0.05 & qRT-PCR & 40 & $\mathrm{CSS}^{\mathrm{u}}$ & & 1.22 & $0.52-2.84$ \\
\hline $106 b$ & Yang TS, 2014 [45] & China & $\mathrm{R}$ & Tissue & 120 & None & Median & qRT-PCR & 45 & $\mathrm{OS}^{u}$ & & 1.79 & $1.10-2.90$ \\
\hline 107 & Li X, $2011[46]$ & China & $\mathrm{R}$ & FFPE & 50 & None & 90.95 & qRT-PCR & 48 & $\mathrm{OS}^{\mathrm{u}}$ & & 0.48 & $0.28-0.82$ \\
\hline 107 & Osawa S, 2011 [3] & Japan & $\mathrm{R}$ & FFPE & 37 & II-III & $70 \%$ & qRT-PCR & 60 & $\mathrm{OS}^{\mathrm{u}}$ & & 4.09 & $1.26-13.32$ \\
\hline 107 & Inoue T, 2012 [47] & Japan & $\mathrm{R}$ & Frozen & 161 & I-IV & 2.74 & RT-qPCR & 60 & $\mathrm{OS}^{\mathrm{m}}$ & & 2.21 & $1.18-4.61$ \\
\hline $\begin{array}{l}125 a- \\
3 p\end{array}$ & $\begin{array}{c}\text { Hashiguchi Y, } 2012 \\
\text { [49] }\end{array}$ & Japan & $\mathrm{R}$ & Frozen & 70 & I-IV & 7.42 & RT-qPCR & 147.6 & $\mathrm{OS}^{u}$ & 3.01 & & $1.26-7.20$ \\
\hline $\begin{array}{l}125 a- \\
5 p\end{array}$ & Nishida N, 2011 [50] & Japan & $\mathrm{R}$ & Frozen & 87 & I-IV & None & RT-qPCR & 147.6 & $\mathrm{OS}^{u}$ & 2.16 & & $0.96-4.86$ \\
\hline $125 \mathrm{a}$ & Dai J, 2015 [51] & China & $\mathrm{R}$ & FFPE & 73 & I-IV & None & qRT-PCR & 62 & $\mathrm{OS}^{\mathrm{u}}$ & 1.31 & & $0.54-3.18$ \\
\hline 137 & $\mathrm{Gu} Q, 2015[62]$ & $\begin{array}{l}\text { China } \\
\text { Set I } \\
\text { China } \\
\text { Set II }\end{array}$ & $\mathrm{R}$ & Frozen & $\begin{array}{l}67 \\
87\end{array}$ & I-III & Median & qRT-PCR & 96 & $\begin{array}{l}\mathrm{OS}^{\mathrm{m}} \\
\mathrm{OS}^{\mathrm{m}}\end{array}$ & $\begin{array}{l}6.80 \\
2.41\end{array}$ & & $\begin{array}{c}2.06-22.48 \\
1.13-5.11\end{array}$ \\
\hline 137 & Zheng X, 2015 [63] & China & $\mathrm{R}$ & FFPE & 38 & I-IV & Median & qRT-PCR & 56 & $\mathrm{DFS}^{\mathrm{u}}$ & 2.70 & & $1.18-6.17$ \\
\hline 137 & Du Y, 2016 [64] & China & $\mathrm{R}$ & Tissue & 14 & I-IV & 0.01 & qRT-PCR & 96 & $\mathrm{OS}^{\mathrm{u}}$ & 2.49 & & $0.32-19.59$ \\
\hline 141 & Lu YB, 2015 [65] & China & $\mathrm{R}$ & Frozen & 95 & I-IV & Median & qRT-PCR & 60 & $\mathrm{OS}^{\mathrm{m}}$ & 2.97 & & $1.30-10.00$ \\
\hline 141 & Zhou X, 2015 [66] & China & $\mathrm{R}$ & Frozen & 63 & IIB-IV & Median & qRT-PCR & $>30$ & $\mathrm{DFS}^{u}$ & 2.47 & & $1.22-5.00$ \\
\hline 141 & Huang M, 2016 [67] & China & $\mathrm{R}$ & Frozen & 30 & I-IV & None & qRT-PCR & 26.83 & $\mathrm{OS}^{\mathrm{u}}$ & 2.23 & & $1.04-4.79$ \\
\hline 143 & Osawa S, 2011 [3] & Japan & $\mathrm{R}$ & FFPE & 37 & II-III & $70 \%$ & qRT-PCR & 60 & $\mathrm{OS}^{\mathrm{u}}$ & & 2.95 & $0.19-46.23$ \\
\hline 143 & Naito Y, 2014 [69] & Japan & $\mathrm{R}$ & Frozen & 66 & I-IV & $1 / 3$ & qRT-PCR & 50 & $\mathrm{CSS}^{\mathrm{m}}$ & & 2.62 & $1.21-5.80$ \\
\hline
\end{tabular}

(Continued) 


\begin{tabular}{|c|c|c|c|c|c|c|c|c|c|c|c|c|c|}
\hline miRNA & Study & Country & $\begin{array}{l}\text { Study } \\
\text { design }\end{array}$ & Sample & Number & Stage & Cut-off & Method & $\begin{array}{l}\text { Follow-up } \\
\text { (month) }\end{array}$ & Result & $\begin{array}{c}\text { HR } \\
(\mathrm{L} / \mathrm{H})\end{array}$ & $\begin{array}{c}\text { HR } \\
(H / L)\end{array}$ & $95 \% \mathrm{CI}$ \\
\hline 143 & Li JH, 2016 [70] & China & $\mathrm{R}$ & Frozen & 44 & I-IV & 1.18 & qRT-PCR & 26 & $\mathrm{OS}^{\mathrm{u}}$ & & 0.40 & $0.23-0.70$ \\
\hline $145-5 p$ & Zhang Y, 2016 [72] & China & $\mathrm{R}$ & Frozen & 145 & I-IV & None & RT-qPCR & 66 & $\mathrm{OS}^{\mathrm{m}}$ & 3.87 & & $1.13-11.44$ \\
\hline $145-5 p$ & Li CY, 2017 [56] & TCGA & $\mathrm{R}$ & Tissue & 361 & I-IV & None & Downloaded & 60 & $\mathrm{OS}^{\mathrm{u}}$ & 1.37 & & $1.08-1.74$ \\
\hline 145 & Naito Y, 2014 [73] & Japan & $\mathrm{R}$ & FFPE & 71 & I-IV & Median & qRT-PCR & 66.67 & $\mathrm{CSS}^{\mathrm{m}}$ & 0.71 & & $0.33-1.49$ \\
\hline 145 & Liu HT, 2017 [34] & China & $\mathrm{R}$ & FFPE & 102 & I-IV & Median & RT-qPCR & 67 & $\mathrm{OS}^{\mathrm{u}}$ & 1.68 & & $0.87-3.25$ \\
\hline $146 \mathrm{a}$ & Kogo R, 2011 [74] & Japan & $\mathrm{R}$ & Frozen & 90 & I-IV & Median & qRT-PCR & 132 & $\mathrm{OS}^{\mathrm{u}}$ & 2.20 & & $1.31-3.70$ \\
\hline $146 a$ & Hou Z, 2012 [75] & China & $\mathrm{R}$ & FFPE & 30 & I-IV & 0.34 & qRT-PCR & 36 & $\mathrm{OS}^{\mathrm{u}}$ & 2.59 & & $1.24-5.39$ \\
\hline $146 \mathrm{a}$ & Luo Z, 2017 [76] & China & $\mathrm{R}$ & Frozen & 93 & III-IV & ROC & RT-qPCR & 72 & $\mathrm{OS}^{\mathrm{u}}$ & 7.75 & & $1.66-35.71$ \\
\hline $150-5 p$ & Yoon SO, 2016 [77] & Korea & $\mathrm{R}$ & FFPE & $\begin{array}{l}140 \\
118\end{array}$ & I-IV & 2.00 & RT-qPCR & 101.8 & $\begin{array}{l}\mathrm{OS}^{\mathrm{m}} \\
\mathrm{RFS}^{\mathrm{u}}\end{array}$ & & $\begin{array}{l}0.88 \\
1.84\end{array}$ & $\begin{array}{l}0.37-2.09 \\
0.98-3.43\end{array}$ \\
\hline 150 & Katada T, 2009 [12] & Japan & $\mathrm{R}$ & Frozen & 42 & None & None & qRT-PCR & 60 & $\mathrm{OS}^{\mathrm{m}}$ & & 6.10 & $0.76-50.00$ \\
\hline 150 & Smid D, 2016 [79] & Czech & $\mathrm{R}$ & FFPE & $\begin{array}{l}41 \\
40\end{array}$ & None & $\begin{array}{l}6.00 \\
6.70\end{array}$ & qRT-PCR & $>100$ & $\begin{array}{l}\mathrm{OS}^{\mathrm{u}} \\
\mathrm{PFS}^{\mathrm{u}}\end{array}$ & & $\begin{array}{l}1.91 \\
2.08\end{array}$ & $\begin{array}{l}1.14-3.21 \\
1.11-3.91\end{array}$ \\
\hline $183-5 p$ & Li CY, 2017 [56] & TCGA & $\mathrm{R}$ & Tissue & 361 & I-IV & None & Downloaded & 60 & $\mathrm{OS}^{\mathrm{u}}$ & 0.64 & & $0.47-0.87$ \\
\hline 183 & Cao LL, 2014 [83] & China & $\mathrm{R}$ & Frozen & 52 & I-IV & 3.55 & qRT-PCR & 60 & $\mathrm{OS}^{\mathrm{u}}$ & 2.83 & & $1.31-6.10$ \\
\hline 183 & Xu L, 2014 [84] & China & $\mathrm{R}$ & Tissue & 65 & I-IV & Median & RT-qPCR & 102 & $\mathrm{OS}^{\mathrm{u}}$ & 1.94 & & $1.11-3.39$ \\
\hline 192 & Chen Q, 2014 [48] & China & $\mathrm{R}$ & Plasma & 61 & III-IV & 2.00 & qRT-PCR & 43 & $\mathrm{OS}^{\mathrm{m}}$ & & 0.89 & $0.39-2.04$ \\
\hline 192 & Xu YJ, 2015 [87] & China & $\mathrm{R}$ & Frozen & 38 & I-IV & None & qRT-PCR & 81 & $\mathrm{OS}^{\mathrm{u}}$ & & 0.99 & $0.96-1.02$ \\
\hline 192 & Smid D, 2016 [79] & Czech & $\mathrm{R}$ & FFPE & 41 & None & 2.30 & qRT-PCR & $>100$ & $\mathrm{OS}^{\mathrm{u}}$ & & 7.43 & $2.71-20.41$ \\
\hline $196 \mathrm{a}$ & Sun M, 2012 [90] & China & $\mathrm{R}$ & Frozen & 31 & II-IV & 40.90 & RT-qPCR & 36 & $\mathrm{OS}^{\mathrm{u}}$ & & 4.19 & $1.78-9.83$ \\
\hline $196 \mathrm{a}$ & Mu YP, 2014 [88] & China & $\mathrm{R}$ & Frozen & 48 & I-IV & 5.69 & qRT-PCR & 60 & $\mathrm{OS}^{\mathrm{u}}$ & & 2.88 & $1.43-5.79$ \\
\hline $196 \mathrm{a}$ & Tsai MM, 2014 [91] & China & $\mathrm{R}$ & Tissue & 109 & I-IV & 77.30 & qRT-PCR & 60 & $\mathrm{OS}^{\mathrm{u}}$ & & 2.27 & $1.50-3.43$ \\
\hline $196 \mathrm{a}$ & Tsai MM, 2016 [92] & China & $\mathrm{R}$ & Plasma & 98 & I-IV & 1.15 & qRT-PCR & 72 & $\mathrm{OS}^{\mathrm{m}}$ & & 3.06 & $1.10-8.50$ \\
\hline $\begin{array}{l}196 b- \\
5 p\end{array}$ & Li CY, 2017 [56] & TCGA & $\mathrm{R}$ & Tissue & 361 & I-IV & None & Downloaded & 60 & $\mathrm{OS}^{\mathrm{u}}$ & & 2.07 & $1.37-3.13$ \\
\hline $196 b$ & Lim JY, 2013 [93] & $\begin{array}{l}\text { South } \\
\text { Korea }\end{array}$ & $\mathrm{R}$ & Frozen & 57 & I-IV & None & qRT-PCR & 75 & $\mathrm{OS}^{\mathrm{u}}$ & & 1.50 & $1.06-2.12$ \\
\hline $196 b$ & Tsai MM, 2014 [91] & China & $\mathrm{R}$ & Tissue & 109 & I-IV & 21.70 & qRT-PCR & 60 & $\mathrm{OS}^{\mathrm{u}}$ & & 1.55 & $1.16-2.06$ \\
\hline $196 b$ & Tsai MM, 2016 [92] & China & $\mathrm{R}$ & Plasma & 98 & I-IV & 0.93 & qRT-PCR & 72 & $\mathrm{OS}^{\mathrm{m}}$ & & 2.91 & $1.04-8.17$ \\
\hline $200 \mathrm{c}$ & $\begin{array}{l}\text { Valladares-Ayerbes } \\
\text { M, } 2012 \text { [98] }\end{array}$ & Spain & $\mathrm{R}$ & Blood & 52 & I-IV & 62.4 & qRT-PCR & 54 & $\begin{array}{l}\mathrm{OS}^{\mathrm{m}} \\
\mathrm{PFS}^{\mathrm{m}}\end{array}$ & $\begin{array}{l}0.45 \\
0.44\end{array}$ & & $\begin{array}{l}0.22-0.92 \\
0.21-0.92\end{array}$ \\
\hline $200 \mathrm{c}$ & Tang H, 2013 [96] & China & $\mathrm{R}$ & Tissue & 126 & I-IV & 2.00 & qRT-PCR & 58 & $\begin{array}{c}\mathrm{OS}^{\mathrm{u}} \\
\mathrm{DFS}^{\mathrm{u}}\end{array}$ & $\begin{array}{l}2.29 \\
1.83\end{array}$ & & $\begin{array}{l}1.38-3.81 \\
1.15-2.92\end{array}$ \\
\hline $200 \mathrm{c}$ & Zhang HP, 2015 [99] & China & $\mathrm{R}$ & Serum & 98 & I-IV & Median & qRT-PCR & 60 & $\mathrm{OS}^{\mathrm{m}}$ & 0.25 & & $0.10-0.37$ \\
\hline $200 \mathrm{c}$ & Zhou X, 2015 [66] & China & $\mathrm{R}$ & Frozen & 63 & IIB-IV & Median & qRT-PCR & $>30$ & DFS $^{u}$ & 1.70 & & $1.21-2.38$ \\
\hline 206 & Yang Q, 2013 [104] & China & $\mathrm{R}$ & Tissue & 98 & I-IV & 2.40 & RT-qPCR & 139 & $\mathrm{OS}^{\mathrm{m}}$ & 2.56 & & $1.13-5.82$ \\
\hline 206 & Shi H, 2015 [105] & China & $\mathrm{R}$ & Frozen & 220 & I-IV & Median & qRT-PCR & 60 & $\mathrm{OS}^{\mathrm{m}}$ & 6.82 & & $1.51-21.29$ \\
\hline 206 & Hou CG, 2016 [106] & China & $\mathrm{R}$ & Serum & 150 & I-III & Median & RT-qPCR & 60 & $\mathrm{OS}^{\mathrm{m}}$ & 2.39 & & $1.16-4.91$ \\
\hline 214 & Ueda T, 2010 [1] & Japan & $\mathrm{R}$ & Frozen & 101 & I-IV & None & qRT-PCR & 102.33 & $\mathrm{OS}^{\mathrm{m}}$ & & 2.70 & $1.30-5.61$ \\
\hline 214 & Yang TS, 2013 [110] & China & $\mathrm{R}$ & Frozen & 120 & I-IV & None & qRT-PCR & 45 & $\mathrm{OS}^{\mathrm{u}}$ & & 1.77 & $1.06-2.96$ \\
\hline 214 & $\begin{array}{c}\text { Wang YW, } 2014 \\
{[111]}\end{array}$ & China & $\mathrm{R}$ & FFPE & 80 & I-IV & Median & RT-qPCR & 72 & $\mathrm{OS}^{\mathrm{u}}$ & & 1.20 & $0.67-2.15$ \\
\hline 214 & Liu HT, 2017 [34] & China & $\mathrm{R}$ & FFPE & 102 & I-IV & Median & RT-qPCR & 67 & $\mathrm{OS}^{\mathrm{m}}$ & & 2.75 & $1.12-6.76$ \\
\hline 218 & Tie J, 2010 [114] & China & $\mathrm{R}$ & Frozen & 40 & I-IV & 13.81 & qRT-PCR & 72 & $\mathrm{OS}^{\mathrm{u}}$ & 2.33 & & $1.40-3.89$ \\
\hline 218 & Xin SY, 2014 [115] & China & $\mathrm{R}$ & Serum & 68 & I-IV & None & qRT-PCR & 36 & $\mathrm{OS}^{\mathrm{m}}$ & 3.16 & & $1.06-9.40$ \\
\hline
\end{tabular}

(Continued) 


\begin{tabular}{|c|c|c|c|c|c|c|c|c|c|c|c|c|c|}
\hline miRNA & Study & Country & $\begin{array}{l}\text { Study } \\
\text { design }\end{array}$ & Sample & Number & Stage & Cut-off & Method & $\begin{array}{l}\text { Follow-up } \\
\text { (month) }\end{array}$ & Result & $\underset{(\mathrm{L} / \mathrm{H})}{\mathrm{HR}}$ & $\begin{array}{c}\text { HR } \\
(\mathrm{H} / \mathrm{L})\end{array}$ & $95 \% \mathrm{CI}$ \\
\hline 218 & $\begin{array}{c}\text { Wang XX, } 2016 \\
{[116]}\end{array}$ & China & $\mathrm{R}$ & Tissue & 112 & I-IV & Median & qRT-PCR & 60 & $\mathrm{OS}^{\mathrm{m}}$ & 3.19 & & $1.55-8.37$ \\
\hline 335 & Yan Z, 2012 [127] & China & $\mathrm{R}$ & Both & 74 & I-IV & None & RT-qPCR & 108 & $\mathrm{OS}^{\mathrm{u}}$ & 0.14 & & $0.04-0.49$ \\
\hline 335 & Yang B, 2016 [41] & China & $\mathrm{R}$ & Tissue & 50 & I-IV & Median & qRT-PCR & 60 & $\mathrm{OS}^{\mathrm{u}}$ & 4.88 & & $1.90-12.55$ \\
\hline 335 & $\begin{array}{c}\text { Zhang JK, } 2017 \\
{[128]}\end{array}$ & China & $\mathrm{R}$ & Frozen & 221 & I-IV & Median & qRT-PCR & 60 & $\mathrm{DFS}^{u}$ & 1.65 & & $1.11-2.45$ \\
\hline 451 & Ren C, 2016 [4] & China & $\mathrm{R}$ & FFPE & 180 & I-IV & None & ISH & 97.2 & $\mathrm{OS}^{\mathrm{m}}$ & 2.01 & & $1.36-2.96$ \\
\hline 451 & $\begin{array}{c}\text { Bandres E, } 2009 \\
{[141]}\end{array}$ & Spain & $\mathrm{R}$ & FFPE & 45 & I-III & Median & qRT-PCR & 172 & $\begin{array}{c}\mathrm{OS}^{\mathrm{u}} \\
\mathrm{DFS}^{\mathrm{m}}\end{array}$ & $\begin{array}{l}2.02 \\
3.70\end{array}$ & & $\begin{array}{l}0.76-5.38 \\
1.57-8.70\end{array}$ \\
\hline 451 & $\begin{array}{c}\text { Brenner B, } 2011 \\
{[142]}\end{array}$ & Israel & $\mathrm{R}$ & FFPE & 45 & I-III & Median & qRT-PCR & 50 & $\mathrm{RFS}^{u}$ & 0.05 & & $0.01-0.29$ \\
\hline 451 & Su Z, 2015 [143] & China & $\mathrm{R}$ & FFPE & 107 & I-IV & Mean & qRT-PCR & 72 & $\mathrm{OS}^{\mathrm{u}}$ & 1.08 & & $0.53-2.19$ \\
\hline $486-5 p$ & Li CY, 2017 [56] & TCGA & $\mathrm{R}$ & Tissue & 361 & I-IV & None & Downloaded & 60 & $\mathrm{OS}^{\mathrm{u}}$ & 1.85 & & $1.22-2.81$ \\
\hline $486-5 p$ & Chen H, 2015 [147] & China & $\mathrm{R}$ & FFPE & 84 & I-IV & None & ISH & 75 & $\mathrm{OS}^{\mathrm{m}}$ & 3.61 & & $1.99-6.54$ \\
\hline $486-5 p$ & Ren C, 2016 [148] & China & $\mathrm{R}$ & FFPE & 84 & I-IV & None & ISH & 93.6 & $\mathrm{OS}^{\mathrm{m}}$ & 2.55 & & $1.39-4.69$ \\
\hline 506 & Deng J, 2015 [154] & China & $\mathrm{R}$ & Frozen & 63 & None & None & qRT-PCR & $>60$ & $\mathrm{OS}^{\mathrm{u}}$ & 3.05 & & $1.19-7.79$ \\
\hline 506 & Li Z, 2015 [155] & China & $\mathrm{R}$ & Frozen & 84 & I-IV & Mean & qRT-PCR & $>60$ & $\mathrm{OS}^{\mathrm{u}}$ & 1.76 & & $0.73-4.27$ \\
\hline 506 & $\begin{array}{c}\text { Sakimura S, } 2015 \\
{[156]}\end{array}$ & Japan & $\mathrm{R}$ & Tissue & 141 & I-IV & Median & qRT-PCR & $>140$ & $\mathrm{OS}^{\mathrm{m}}$ & 1.90 & & $1.05-3.59$ \\
\hline
\end{tabular}

HR (L/H): hazard ratios of low expression versus high expression of miRNAs; HR (H/L): hazard ratios of high expression versus low expression of miRNAs; CI: confidence intervals; TCGA: The Cancer Genome Atlas; R: retrospective; P: prospective; FFPE: formalin-fixed paraffin-embedded; ROC: receiver operating characteristic; qRT-PCR: quantitative real-time polymerase chain reaction; RT-qPCR: reverse transcription quantitative real-time polymerase chain reaction; IHC: immunohistochemistry; ISH: in-situ hybridization; OS: overall survival; RFS: recurrence-free survival; CSS: cause-specific survival; DFS: disease-free survival; PFS: progression-free survival; "Univariate analysis; ${ }^{m}$ Multivariate analysis. In order to facilitate read and statistics, studies estimating prognostic value of different miRNAs are shown in blue and white; studies which cannot be merged are shown in yellow.

\section{Meta-analysis}

A summary of the HR evaluated from the whole combined analysis for all the miRNAs was shown in Table 3.

\section{High expression of miR-21 predicts poor OS}

Five studies $[3,15,16,18,19]$ analyzed associations between high expression of miR-21 and OS, indicating that GC patients with high miR-21 expression had a significantly shorter OS than those with low miR-21 expression $(\mathrm{HR}=1.77,95 \% \mathrm{CI}=1.01-3.08, \mathrm{P}<0.05$, Figure $2 \mathrm{~A})$.

\section{No significant association between high expression of miR-21 and RFS/CSS}

Three researches $[6,14,17]$ focused on connections between high expression of miR-21 and RFS/CSS, suggesting that there was no significant association between high expression of miR-21 and RFS/CSS $(\mathrm{HR}=2.10,95 \% \mathrm{CI}=0.72-6.12, \mathrm{P}=0.17$, Figure $2 \mathrm{~A})$.

\section{Publication bias}

In order to evaluate publication bias for OS of GC patients with high miR-21 expression, the Begg's funnel plot was used by us (Figure 2B). And the P value was 0.62 , indicating absence of publication bias.

\section{Sensitivity analysis}

During the study about OS of GC patients with high miR-21 expression, our sensitivity analysis did not indicate alterations in the results according to the exclusion of any individual study (Figure 2C), suggesting that no single research significantly influenced the pooled HR and the $95 \% \mathrm{CI}$.

\section{No significant association between low expression of miR-34a and OS or OS (multivariate analysis)}

There was no significant association between low expression of miR-34a and $\mathrm{OS}(\mathrm{HR}=1.25,95 \% \mathrm{CI}=0.59$ $2.65, \mathrm{P}=0.56$, Figure $2 \mathrm{D}$ ) or $\mathrm{OS}$ (multivariate analysis, $\mathrm{HR}=1.56,95 \% \mathrm{CI}=0.95-2.55, \mathrm{P}=0.08$, Figure 2D).

GC patients with high expression of miR-20b, 106b, 196a, 196b, 214 or low expression of miR125a, 137, 141, 145, 146a, 206, 218, 451, 486-5p, 506 have a significantly poor OS

The details were shown in Table 3 and Figures 3-8. 
Table 3: Summary of the HR for miRNA expression in gastric cancer

\begin{tabular}{|c|c|c|c|c|c|c|c|c|c|}
\hline miRNA & $\begin{array}{l}\text { Survival } \\
\text { analysis }\end{array}$ & $\begin{array}{l}\text { Number } \\
\text { of articles }\end{array}$ & $\begin{array}{l}\text { Included } \\
\text { references }\end{array}$ & HR & $95 \%$ CI & Figure & P value & $\begin{array}{c}\text { Heterogeneity } \\
\text { (Higgins } I^{2} \text { statistic) }\end{array}$ & $\begin{array}{c}\text { Total } \\
\text { patients }\end{array}$ \\
\hline High miR-20a & OS & 3 & $3,5,11$ & 1.25 & $0.84-1.87$ & 3 & 0.27 & $\mathrm{I}^{2}=70.7 \%, \mathrm{P}=0.03$ & 199 \\
\hline High miR-20b & OS & 3 & $3,12,13$ & 2.38 & $1.16-4.87$ & 3 & 0.02 & $\mathrm{I}^{2}=0.0 \%, \mathrm{P}=0.60$ & 178 \\
\hline High miR-21 & RFS/CSS & 3 & $6,14,17$ & 2.10 & $0.72-6.12$ & $2 \mathrm{~A}$ & 0.17 & $\mathrm{I}^{2}=65.6 \%, \mathrm{P}=0.06$ & 180 \\
\hline High miR-21 & OS & 5 & $3,15,16,18,19$ & 1.77 & $1.01-3.08$ & $2 \mathrm{~A}$ & $<0.05$ & $\mathrm{I}^{2}=57.8 \%, \mathrm{P}=0.05$ & 327 \\
\hline Low miR-27b & OS & 3 & $32-34$ & 1.18 & $0.75-1.85$ & 3 & 0.47 & $\mathrm{I}^{2}=36.1 \%, \mathrm{P}=0.21$ & 319 \\
\hline Low miR-34a & OS & 5 & $3,38-41$ & 1.25 & $0.59-2.65$ & $2 \mathrm{D}$ & 0.56 & $\mathrm{I}^{2}=68.4 \%, \mathrm{P}=0.13$ & 457 \\
\hline Low miR-34a & $\mathrm{OS}^{\mathrm{m}}$ & 2 & 38,40 & 1.56 & $0.95-2.55$ & $2 \mathrm{D}$ & 0.08 & $\mathrm{I}^{2}=51.0 \%, \mathrm{P}=0.15$ & 213 \\
\hline High miR-106b & OS & 2 & 3,45 & 1.84 & $1.15-2.94$ & 3 & 0.01 & $\mathrm{I}^{2}=0.0 \%, \mathrm{P}=0.67$ & 157 \\
\hline High miR-107 & OS & 3 & $3,46,47$ & 1.52 & $0.42-5.57$ & 3 & 0.52 & $\mathrm{I}^{2}=88.8 \%, \mathrm{P}<0.01$ & 248 \\
\hline Low miR-125a & OS & 3 & $49-51$ & 2.06 & $1.26-3.37$ & 4 & $<0.01$ & $\mathrm{I}^{2}=0.0 \%, \mathrm{P}=0.42$ & 230 \\
\hline Low miR-137 & OS & 2 & 62,64 & 3.21 & $1.68-6.13$ & 4 & $<0.01$ & $\mathrm{I}^{2}=6.0 \%, \mathrm{P}=0.35$ & 168 \\
\hline Low miR-141 & OS & 2 & 65,67 & 2.47 & $1.34-4.56$ & 4 & $<0.01$ & $\mathrm{I}^{2}=0.0 \%, \mathrm{P}=0.66$ & 125 \\
\hline High miR-143 & OS & 2 & 3,70 & 0.68 & $0.12-3.81$ & 4 & 0.66 & $\mathrm{I}^{2}=48.8 \%, \mathrm{P}=0.16$ & 81 \\
\hline Low miR-145 & OS & 3 & $34,56,72$ & 1.62 & $1.07-2.46$ & 4 & 0.02 & $\mathrm{I}^{2}=36.9 \%, \mathrm{P}=0.21$ & 608 \\
\hline Low miR-146a & OS & 3 & $74-76$ & 2.60 & $1.63-4.13$ & 5 & $<0.01$ & $\mathrm{I}^{2}=14.1 \%, \mathrm{P}=0.31$ & 213 \\
\hline High miR-150 & OS & 3 & $12,77,79$ & 1.63 & $0.77-3.45$ & 5 & 0.20 & $\mathrm{I}^{2}=47.8 \%, \mathrm{P}=0.15$ & 223 \\
\hline High miR-150 & RFS/PFS & 2 & 77,79 & 1.96 & $1.25-3.05$ & 5 & $<0.01$ & $\mathrm{I}^{2}=0.0 \%, \mathrm{P}=0.79$ & 158 \\
\hline Low miR-183 & OS & 3 & $56,83,84$ & 1.46 & $0.55-3.83$ & 5 & 0.45 & $\mathrm{I}^{2}=90.2 \%, \mathrm{P}<0.01$ & 478 \\
\hline High miR-192 & OS & 3 & $48,79,87$ & 1.71 & $0.60-4.85$ & 5 & 0.31 & $\mathrm{I}^{2}=87.0 \%, \mathrm{P}<0.01$ & 140 \\
\hline High miR-196a & OS & 4 & $88,90-92$ & 2.66 & $1.94-3.63$ & 6 & $<0.01$ & $\mathrm{I}^{2}=0.0 \%, \mathrm{P}=0.62$ & 286 \\
\hline High miR-196b & OS & 4 & $56,91-93$ & 1.67 & $1.38-2.02$ & 6 & $<0.01$ & $\mathrm{I}^{2}=0.0 \%, \mathrm{P}=0.62$ & 625 \\
\hline Low miR-200c & OS & 3 & $96,98,99$ & 0.65 & $0.16-2.64$ & 6 & 0.54 & $\mathrm{I}^{2}=93.6 \%, \mathrm{P}<0.01$ & 276 \\
\hline Low miR-200c & PFS/DFS & 3 & $66,96,98$ & 1.20 & $0.60-2.38$ & 6 & 0.61 & $\mathrm{I}^{2}=83.1 \%, \mathrm{P}<0.01$ & 241 \\
\hline Low miR-206 & OS & 3 & $104-106$ & 2.85 & $1.73-4.70$ & 7 & $<0.01$ & $\mathrm{I}^{2}=0.0 \%, \mathrm{P}=0.37$ & 468 \\
\hline High miR-214 & OS & 4 & $1,34,110,111$ & 1.84 & $1.27-2.67$ & 7 & $<0.01$ & $\mathrm{I}^{2}=23.0 \%, \mathrm{P}=0.27$ & 403 \\
\hline Low miR-218 & OS & 3 & $114-116$ & 2.61 & $1.74-3.92$ & 7 & $<0.01$ & $\mathrm{I}^{2}=0.0 \%, \mathrm{P}=0.77$ & 220 \\
\hline Low miR-335 & OS & 2 & 41,127 & 0.85 & $\begin{array}{l}0.03- \\
27.50\end{array}$ & 7 & 0.93 & $\mathrm{I}^{2}=94.9 \%, \mathrm{P}<0.01$ & 124 \\
\hline Low miR-451 & OS & 3 & $4,141,143$ & 1.73 & $1.19-2.52$ & 8 & $<0.01$ & $\mathrm{I}^{2}=14.7 \%, \mathrm{P}=0.31$ & 332 \\
\hline Low miR-451 & DFS/RFS & 2 & 141,142 & 0.46 & $\begin{array}{l}0.01- \\
31.06\end{array}$ & 8 & 0.72 & $\mathrm{I}^{2}=95.0 \%, \mathrm{P}<0.01$ & 90 \\
\hline Low miR-486-5p & OS & 3 & $56,147,148$ & 2.45 & $1.65-3.65$ & 8 & $<0.01$ & $\mathrm{I}^{2}=40.0 \%, \mathrm{P}=0.19$ & 529 \\
\hline Low miR-506 & OS & 3 & $154-156$ & 2.07 & $1.33-3.23$ & 8 & $<0.01$ & $\mathrm{I}^{2}=0.0 \%, \mathrm{P}=0.65$ & 288 \\
\hline
\end{tabular}

HR: hazard ratios; CI: confidence intervals; OS: overall survival; RFS: recurrence-free survival; CSS: cause-specific survival; PFS: progression-free survival; DFS: disease-free survival; ' ${ }^{\mathrm{m}}$ Multivariate analysis.

\section{No significant association between high expression of miR-20a, 107, 143, 150, 192 or low expression of miR-27b, 183, 200c, 335 and $O S$}

The details were shown in Table 3 and Figures 3-7.

\section{DISCUSSION}

\section{Present situation}

Increasing evidence has shown that various miRNAs are associated with survival outcome in GC 
patients [1-167]. However, inconsistent results have emerged. For example, expression levels of miR-200c are up-regulated in blood $[98,99]$ but down-regulated [66, 96] in tissue compared with normal samples. Furthermore, expression levels of miR-214 [1, 34, 110, 111] and miR451 [4, 141-143] are unsteadily expressed (up or down). Surprisingly, there are significant associations between aberrant expression levels of them and OS $(\mathrm{P}<0.05$, Table 3 , Figures 7 and 8). Therefore, it is essential to conduct a meta-analysis to better understand associations between expression levels of miRNAs and prognosis of GC patients.

\section{Main findings}

We performed the meta-analyses about 26 miRNAs and OS. As the most studied miRNA, GC patients with high miR-21 expression have a significantly poorer OS than those with low miR-21 expression $(\mathrm{P}<0.05)$. But there is no significant association between high miR21 expression and RFS/CSS $(\mathrm{P}=0.17)$. According to our reference standard, miR-21 is still considered to be a significantly prognostic biomarker. There are some other miRNAs with significantly prognostic value in $\mathrm{GC}$, including miR-20b, 106b, 125a, 137, 141, 145, 146a, 196a, 196b, 206, 214, 218, 451, 486-5p and $506(\mathrm{P}<0.05)$. Among them, miR-20b, 125a, 137, 141, 146a, 196a, 206, $218,486-5$ p and 506 are strong biomarkers of prognosis in $\mathrm{GC}(\mathrm{HR} \geq 2)$.

\section{Molecular mechanisms for studied miRNAs}

In addition to the findings mentioned above, a summary of miRNAs with altered expression, their
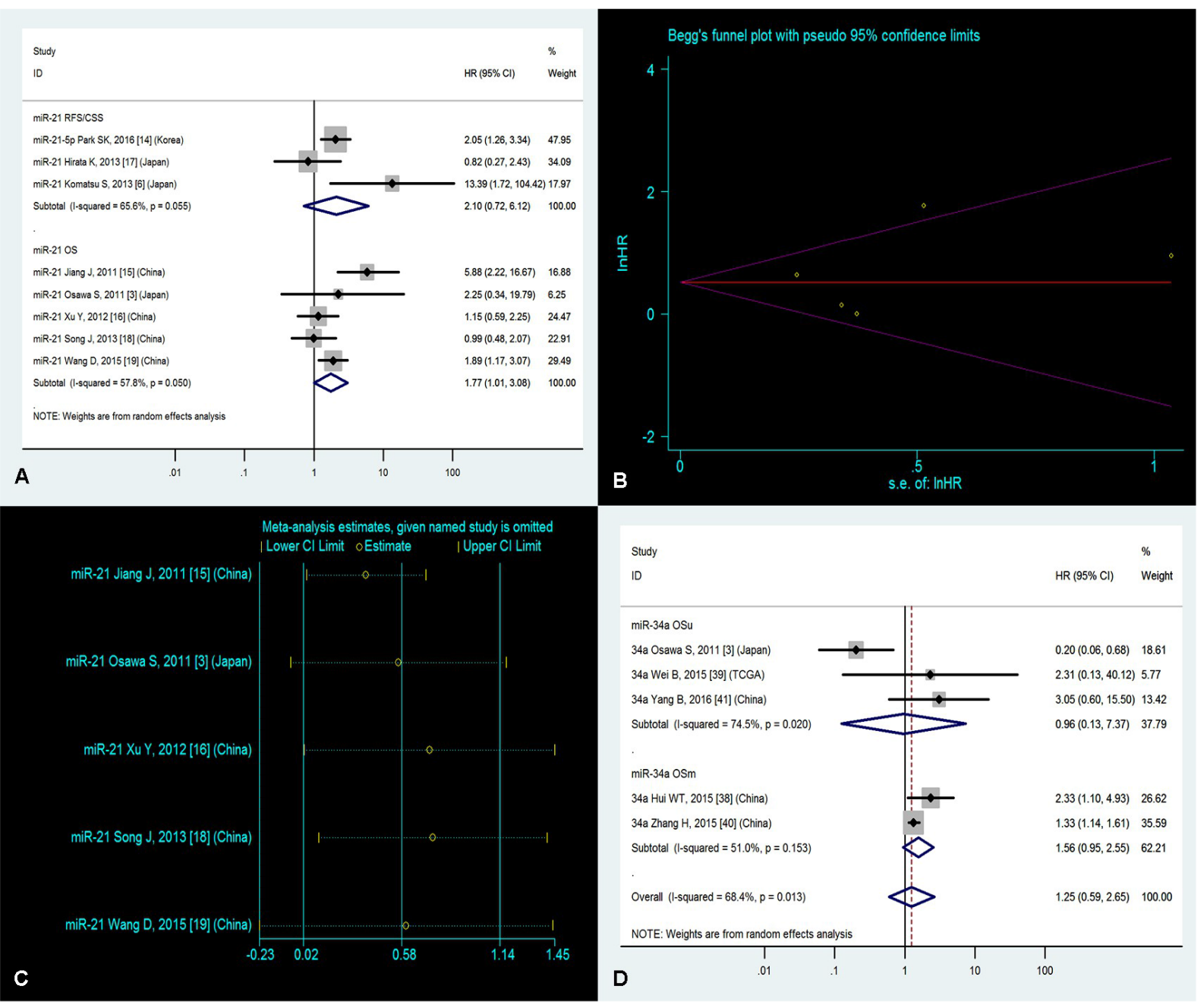

Figure 2: (A) Forest plot of the analyses about high expression of miR-21 and RFS/CSS or OS; (B) Publication bias of the analysis about high expression of miR-21 and OS; (C) Sensitivity analysis of the study about high expression of miR-21 and OS; and (D) Forest plot of the analyses about low expression of miR-34a and OS or OS (multivariate analysis). 
potential targets and pathways entered this study is detailed in Table 4. It is remarkable that there is functional overlapping or connection among those miRNAs. Twenty miRNAs (miR-20a, 27b, 34a, 106b, 107, 125a, 137, 141, 143, 146a, 183, 192, 196a, 196b, 200c, 214, 218, 335, 451 and 506) are involved in cell functions, including cell apoptosis, colony formation, cycle, differentiation and so on. Zhou et al. [66] reported that miR-200c/141 likely increased E-cadherin expression indirectly through down-regulating ZEB1/2, indicating that this pathway may participate in GC migration and invasion. Additionally, Tsai et al. [91] found that GC cell migration and invasion was enhanced by overexpression of miR-196a/-196b and radixin was recognized as a target of miR-196a/-196b.
In a word, these relationships may be involved in the progression of GC.

\section{Strengths of the meta-analysis}

This meta-analysis has several strengths which are as follows: (1) we searched almost all articles with survival outcomes in GC patients with diverse miRNAs. Moreover, the present expression profile of miRNAs was clearly listed in Table 1 in terms of names of miRNAs; (2) articles measuring at least one of survival curves about OS, CSS, DFS, RFS, PFS and MFS were finally included and articles only reporting HR or $95 \%$ CI without any of survival curves were excluded by us; (3) miRNAs

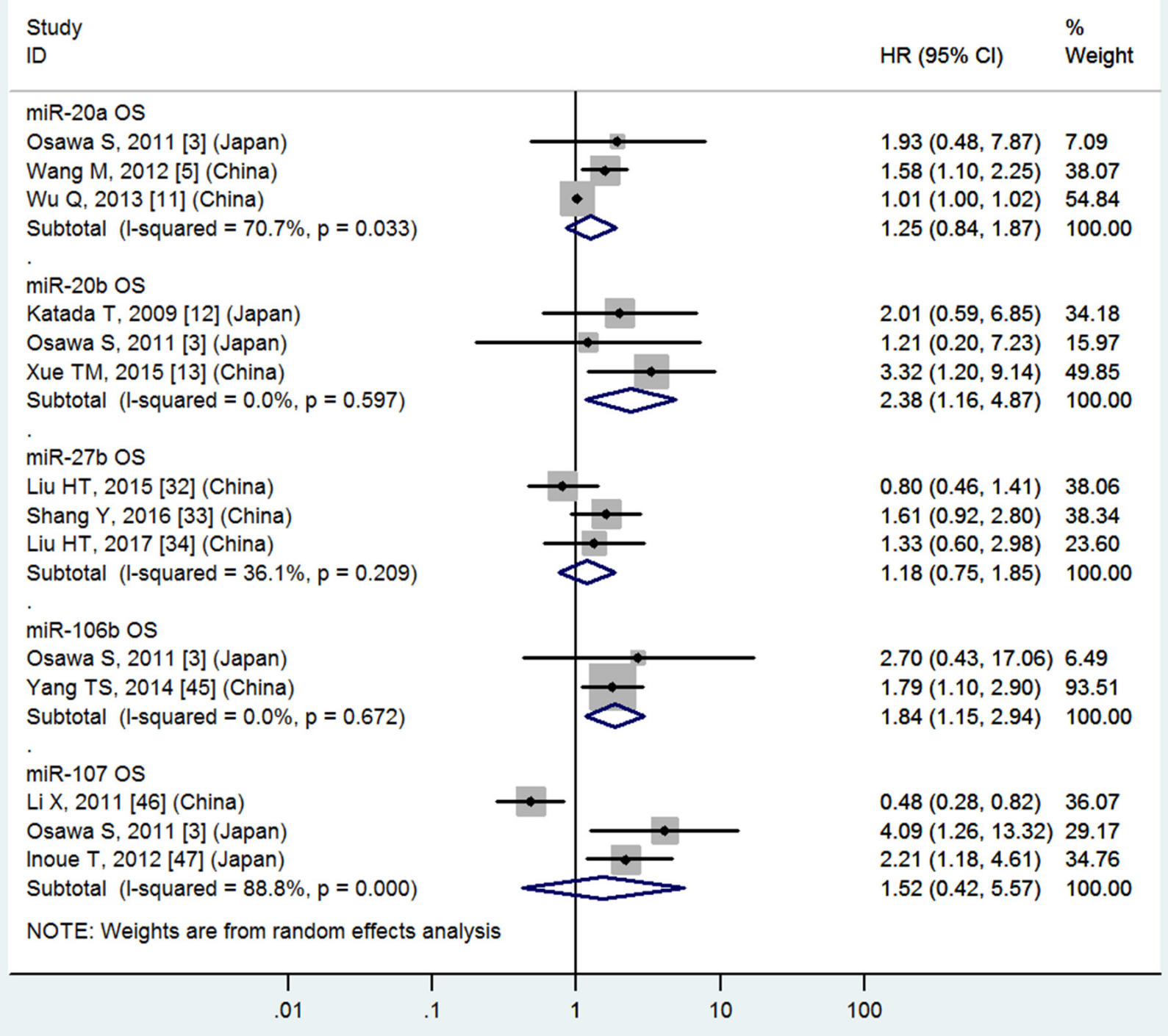

Figure 3: Forest plot of the analyses about high expression of miR-20a, 20b, 106b, 107 or low expression of miR-27b and $\mathrm{OS}$. 
investigated more than or equal to 3 times were conducted meta-analyses; (4) almost all sample sizes of included studies are more than or equal to 30 (except 1 study [64]), enhancing the power and broadening the applicability of the outcomes to GC patients.

\section{Limitations}

However, one should keep in mind the following limitations: (1) 1 miRNA considered as significant biomarker of prognosis contained a high heterogeneity (miR-21); (2) there are many variables among the present meta-analysis, such as different types of samples (tissue, plasma and serum), disease stages, cut-off values and miRNA methods; (3) our meta-analysis only included English articles, which might exclude certain relevant articles with other languages; (4) articles only reporting HR or $95 \%$ CI without survival curves were excluded by us, reducing the sample sizes of included articles; (5) as a result of substantial relevant articles and data about GC, we subjectively and selectively included some researches according to the criteria of inclusion and exclusion (Table 5), leading to ignore a few potential miRNAs with prognostic value.

\section{Implications for future clinical and scientific research}

It is worth mentioning that this meta-analysis is the first systematic estimation of the relevance between miRNA expression and prognosis of GC patients. There are some implications for future clinical and scientific

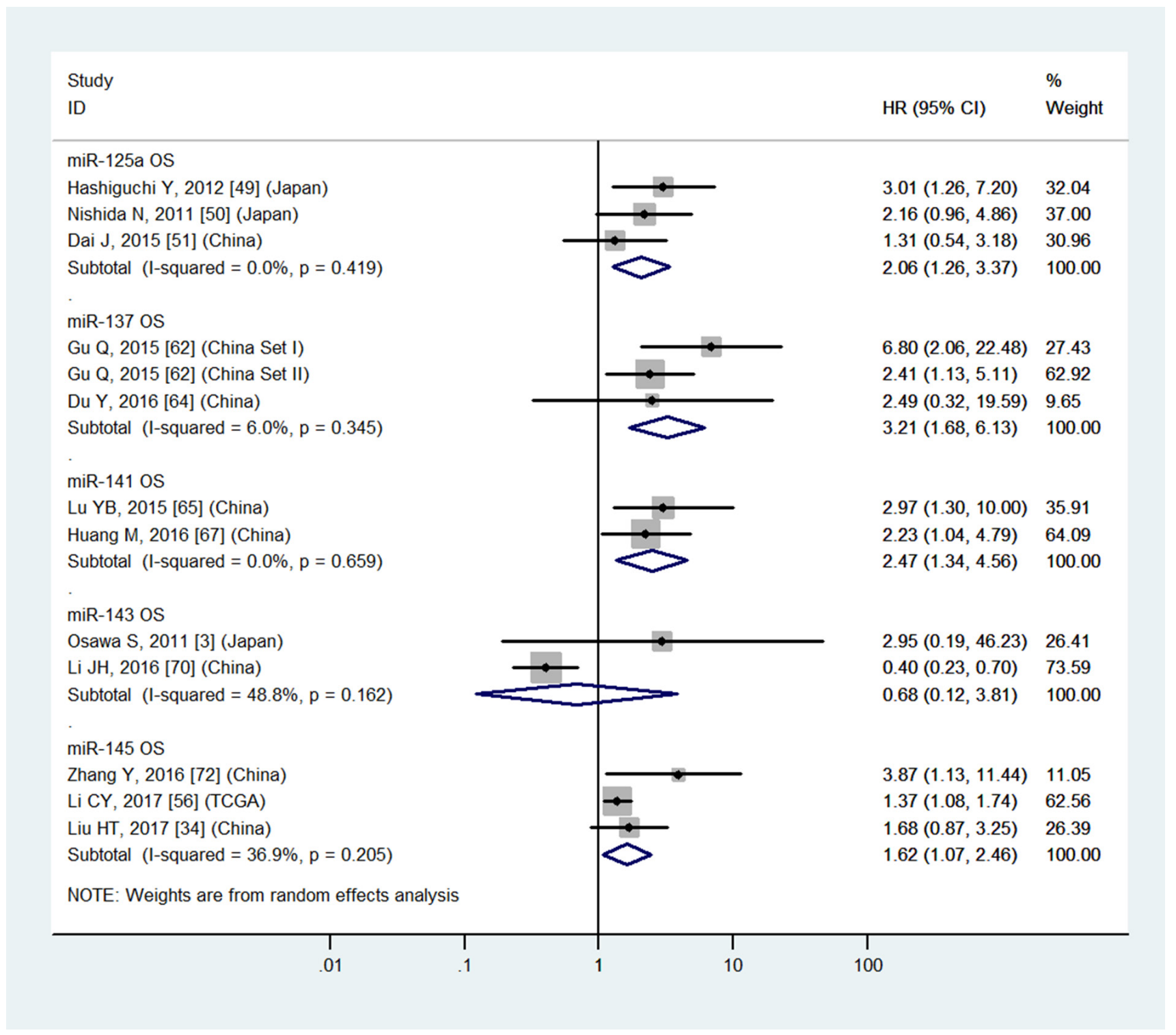

Figure 4: Forest plot of the analyses about high expression of miR-143 or low expression of miR-125a, 137, 141, 145 and $\mathrm{OS}$. 


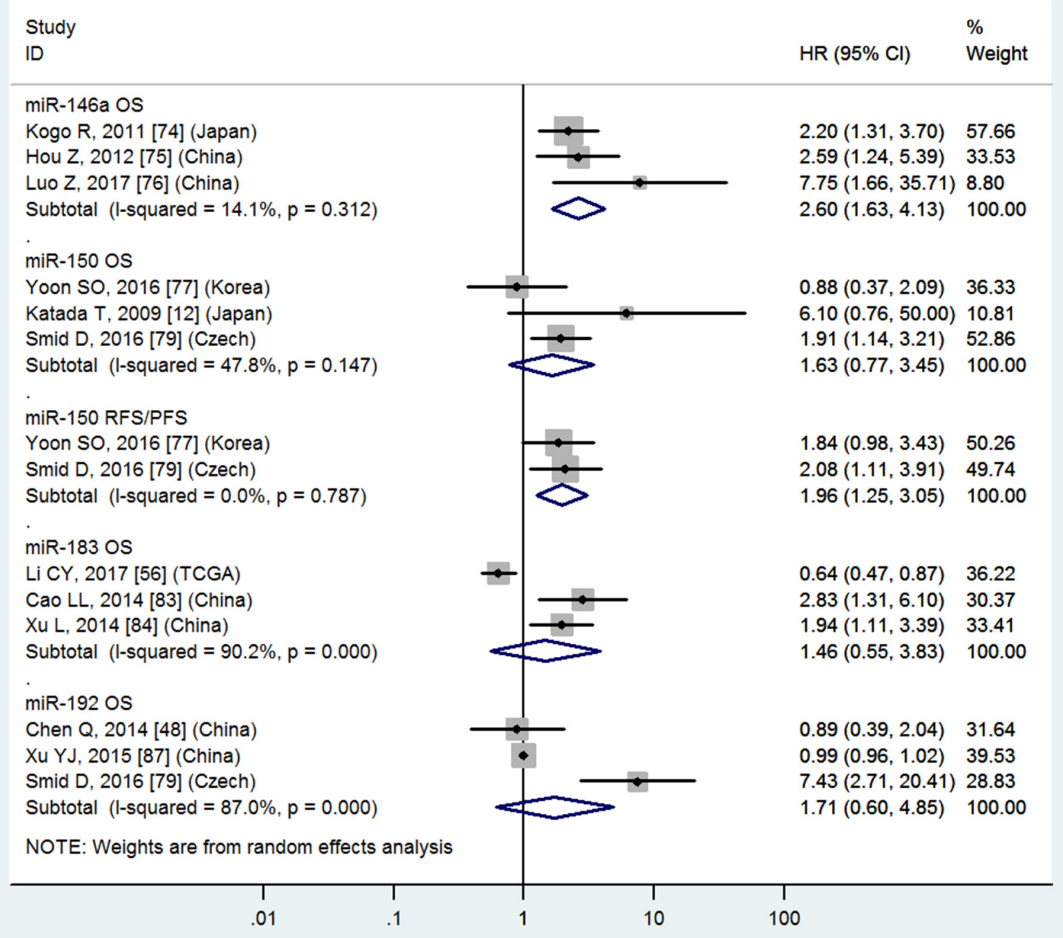

Figure 5: Forest plot of the analyses about high expression of miR-150, 192 or low expression of miR-146a, 183 and OS or RFS/PFS.

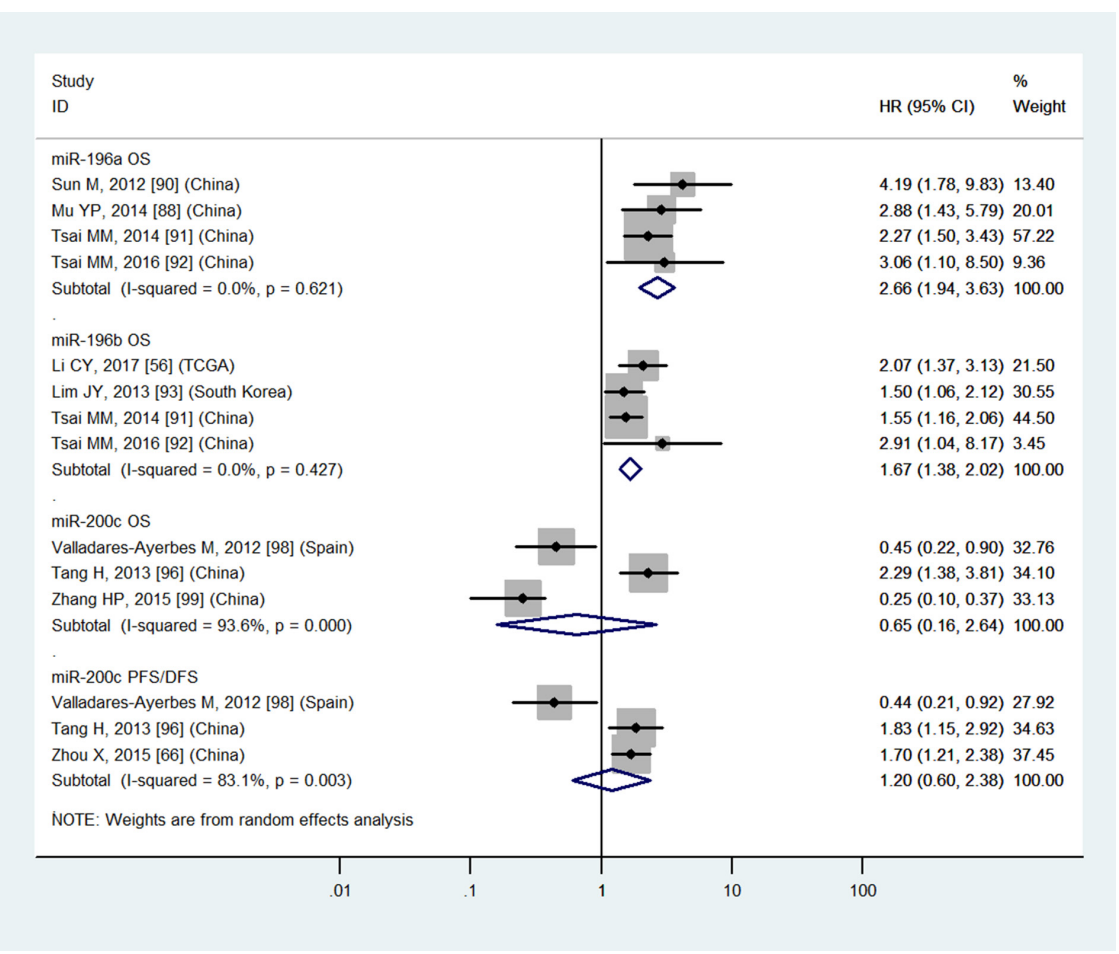

Figure 6: Forest plot of the analyses about high expression of miR-196a, 196b or low expression of miR-200c and OS or PFS/DFS. 


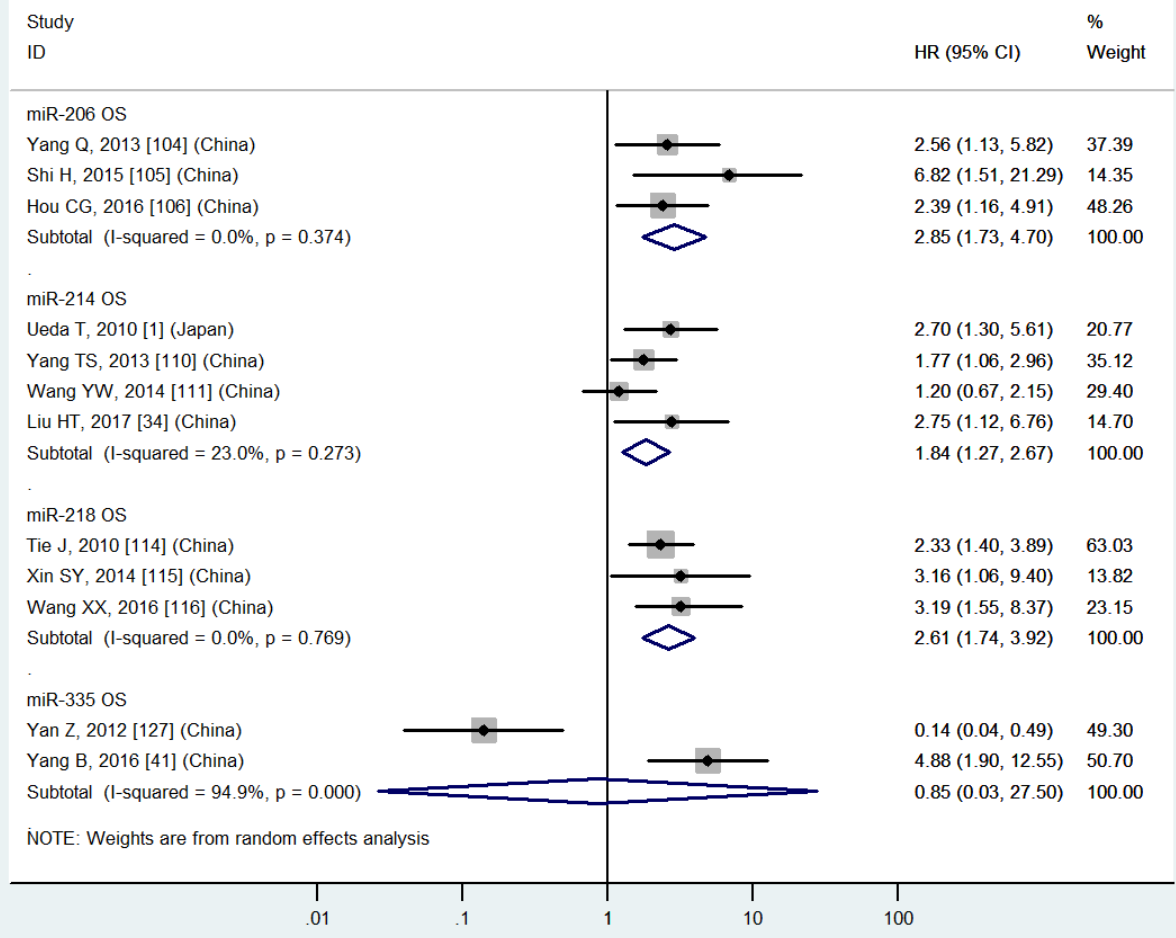

Figure 7: Forest plot of the analyses about high expression of miR-214 or low expression of miR-206, 218, 335 and OS.

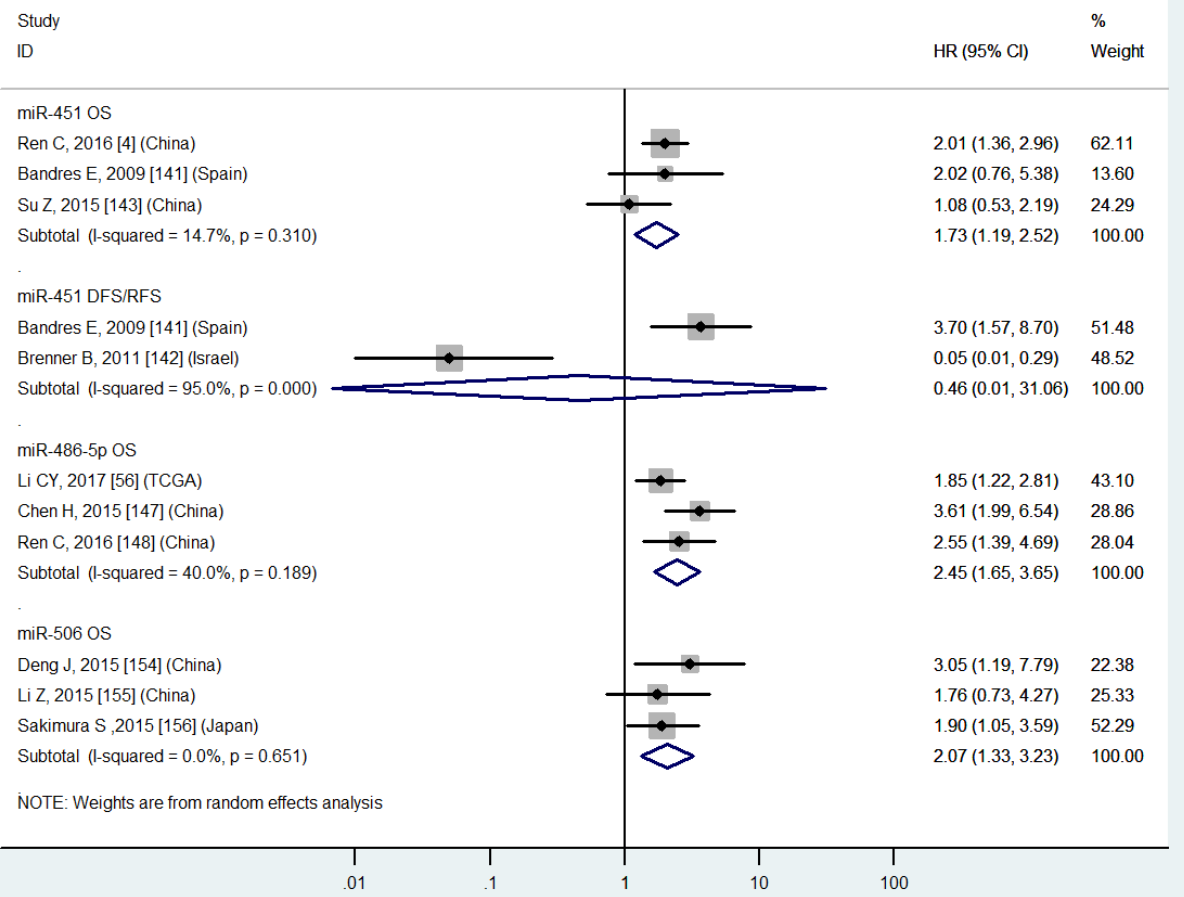

Figure 8: Forest plot of the analyses about low expression of miR-451, 486-5p, 506 and OS or DFS/RFS. 
Table 4: Summary of miRNAs with altered expression, their potential targets and pathways entered this study

\begin{tabular}{|c|c|c|c|c|}
\hline miRNA & Reference & Expression & Potential target & Pathway \\
\hline $20 \mathrm{a}$ & $3,5,11$ & Up & E2F1, HIPK1 & $\begin{array}{l}\text { Cell differentiation, proliferation, self-renewal and } \\
\text { Wnt/ } \beta \text {-catenin signaling }\end{array}$ \\
\hline $20 \mathrm{~b}$ & $3,12,13$ & Up & None & None \\
\hline 21 & $3,6,14-19$ & Up & None & None \\
\hline $27 b$ & $32-34$ & Down & CCNG1, VEGF-C & Cell migration and proliferation \\
\hline $34 \mathrm{a}$ & $3,38-41$ & Down & MET, Survivin & $\begin{array}{l}\text { Cell apoptosis, colony formation, invasion and } \\
\text { proliferation }\end{array}$ \\
\hline $106 \mathrm{~b}$ & $3,6,45$ & Up & PTEN & Cell invasion and migration \\
\hline 107 & $3,46,47$ & Up & DICER1 & Cell invasion and migration \\
\hline $125 \mathrm{a}$ & $49-51$ & Down & VEGF-A, ERBB2 & Cell proliferation \\
\hline 137 & $62-64$ & Down & KLF12, MYO1C, CDK6 & $\begin{array}{l}\text { Cell cycle, differentiation, migration and } \\
\text { proliferation }\end{array}$ \\
\hline 141 & $65-67$ & Down & ZEB1/2, E-cadherin, IGF1R & $\begin{array}{l}\text { Cell colony formation, cycle, invasion, migration, } \\
\text { viability and TGF- } \beta / \text { ZEB signaling }\end{array}$ \\
\hline 143 & $3,69,70$ & Down & BACH1 & $\begin{array}{l}\text { Cell invasion, proliferation and TGF- } \beta / \mathrm{Mad} \\
\text { signaling }\end{array}$ \\
\hline 145 & $34,56,72,73$ & Down & $\alpha-\mathrm{SMA}$ & None \\
\hline $146 \mathrm{a}$ & $74-76$ & Down & EGFR, IRAK1, LIN52 & Cell apoptosis, invasion, migration and proliferation \\
\hline 150 & $12,77,79$ & Up & None & None \\
\hline 183 & $56,83,84$ & Down & EZR, BMI1 & Cell colony formation, invasion and proliferation \\
\hline 192 & $48,79,87$ & Up & None & Cell invasion \\
\hline $196 \mathrm{a}$ & $88,90-92$ & Up & CDKN1B, Rdx & $\begin{array}{l}\text { Cell colony formation, cycle, invasion, migration } \\
\text { and proliferation }\end{array}$ \\
\hline $196 b$ & $56,91-93$ & Up & $\mathrm{Rdx}$ & Cell invasion and migration \\
\hline $200 \mathrm{c}$ & $\begin{array}{l}98,99 \\
66,96\end{array}$ & $\begin{array}{l}\text { Up (blood) } \\
\text { Down (tissue) }\end{array}$ & ZEB1/2, E-cadherin & Cell invasion, migration and TGF- $\beta /$ ZEB signaling \\
\hline 206 & $104-106$ & Down & None & None \\
\hline 214 & $1,34,110,111$ & Up or Down & CSF1, PTEN & Cell invasion, migration and proliferation \\
\hline 218 & $114-116$ & Down & ROBO1 & Cell invasion and sli/ROBO1 signaling \\
\hline 335 & $41,127,128$ & Down & Survivin, BIRC5, CRKL & $\begin{array}{l}\text { Cell apoptosis, cycle, growth, invasion, migration } \\
\text { and proliferaion }\end{array}$ \\
\hline 451 & $4,141-143$ & Up or Down & MIF & Cell invasion, migration and proliferation \\
\hline $486-5 p$ & $56,147-148$ & Down & FGF9 & None \\
\hline 506 & $154-156$ & Down & Yap1, ETS1, SNAI2 & $\begin{array}{l}\text { Cell epithelial-mesenchymal transition, growth, } \\
\text { invasion, migration and proliferation }\end{array}$ \\
\hline
\end{tabular}

E2F1: E2F transcription factor 1; HIPK1: homeodomain interacting protein kinase 1; CCNG1: cyclin G1; VEGF: vascular endothelial growth factor; PTEN: protein tyrosine phosphatase and tensin homologue; DICER1: dicer 1, ribonuclease type III; ERBB2: erb-b2 receptor tyrosine kinase 2; KLF12: krüppel-likefactor 12; MYO1C: myosin 1C; ZEB1/2: zinc finger E-boxbinding homeobox 1/2; IGF1R: insulin-like growth factor 1 receptor; BACH1: BTB domain and CNC homolog 1; $\alpha$-SMA: $\alpha$ smooth muscle actin; EGFR: epidermal growth factor receptor; IRAK1: interleukin 1 receptor associated kinase 1; LIN52: $\operatorname{lin}-52$ homolog (C. elegans); EZR: ezrin; BMI1: BMI1 proto-oncogene, polycomb ring finger; CDKN1B: cyclin dependent kinase inhibitor 1B; Rdx: radixin; CSF1: colony stimulating factor 1; Robo1: roundabout guidance receptor 1; BIRC5: baculoviral IAP repeat containing 5; CRKL: CRK like protoongogene, adaptor protein; MIF: macrophage migration inhibitory factor (glycosylation-inhibiting factor); FGF9: fibroblast growth factor 9; YAP1: Yes associated protein 1; ETS1: ETS proto-oncogene 1, transcription factor; SNAI2: snail family transcriptional repressor 2; TGF- $\beta$ : transforming growth factor- $\beta$; Mad: mothers against dpp; AKT1: AKT serine/threonine kinase 1; sli: slit. 
Table 5: Information of search methods and criteria of inclusion and exclusion

\begin{tabular}{|c|c|}
\hline Methods & Information \\
\hline Search strategy & $\begin{array}{l}4 \text { search engines, including PubMed, EMBASE, Web of } \\
\text { Science and Cochrane Database of Systematic Reviews }\end{array}$ \\
\hline Search deadline & March 19, 2017 \\
\hline Search term & mir and gastric cancer \\
\hline Inclusion criteria & $\begin{array}{l}\text { (1) Patients with gastric cancer; } \\
\text { (2) Expression of miRNAs and survival outcome in } \\
\text { tissue, plasma or serum were measured; } \\
\text { (3) At least, one of survival curves about overall survival } \\
\text { (OS), cause-specific survival (CSS), disease-free survival } \\
\text { (DFS), recurrence-free survival (RFS), progression-free } \\
\text { survival (PFS) and metastasis-free survival (MFS) } \\
\text { was measured, with or without the HR or } 95 \% \mathrm{CI} \text {; } \\
\text { (4) Full text articles in English }\end{array}$ \\
\hline Exclusion criteria & $\begin{array}{l}\text { (1) Reviews, letters or laboratory studies without } \\
\text { original data and retracted articles; } \\
\text { (2) Frequency of studies estimating prognostic value } \\
\text { of miRNAs } \leq 2 \text {; } \\
\text { (3) Studies which cannot be merged; } \\
\text { (4) If more than one article had been published on the } \\
\text { identical study cohort, only the most comprehensive } \\
\text { study was selected for the present meta-analysis }\end{array}$ \\
\hline
\end{tabular}

research in the present meta-analysis: (1) for clinical doctors and other healthcare providers, combined detection of miRNA expression can greatly enhance the estimation about survival time of GC patients and timely treatment can be offered; (2) for scientific researchers, the present study trend on associations between miRNAs and prognosis of GC patients can be conveniently seen in Table 1. As a result, selectively basic experiments can be performed by them (Table 4); (3) inconsistent outcomes of prognosis about miRNAs may be solved according to the basement of the current meta-analysis.

\section{MATERIALS AND METHODS}

\section{Search strategy, inclusion criteria and exclusion criteria}

The details were presented in Table 5. Two authors (Yue Zhang and Dong-Hui Guan) independently performed this comprehensive online search.

\section{Quality assessment}

Yue Zhang and Dong-Hui Guan confirmed all eligible investigations that analyzed the prognostic value of miRNAs in GC, and Yue-Hua Jiang reassessed uncertain data.

\section{Statistical analysis}

All analyses were conducted using Stata version 13.0 (StataCorp, College Station, Texas, USA). The relative effect sizes for $\mathrm{HR}$ were characterized as moderate (protective [0.51-0.75] or contributory [1.351.99]) and large $(\leq 0.50$ or $\geq 2)$. The HR was considered significant at the $\mathrm{P}<0.05$ level if the $95 \% \mathrm{CI}$ did not include the value 1 . If the $\mathrm{P}$ values from $\mathrm{OS}$ and other survival results about corresponding miRNAs were inconsistent, the HR from OS was considered to the main reference standard. Because different types of samples (tissue, plasma and serum) from GC patients at different disease stages, cut-off values and miRNA methods were used in individual studies, random-effects models (DerSimonian-Laird method) were more appropriate than fixed-models (Mantel-Haenszel method) for most of the analyses. Consequently, the random-effects models were used in the current meta-analysis. Publication bias was estimated using the Begg's funnel plot. A two-tailed $\mathrm{P}$ value $<0.05$ was considered significant. Sensitivity analysis (influence analysis) was carried out to test how powerful the combined effect size was to removal of individual investigations. If the point assessment was out the $95 \% \mathrm{CI}$ of the pooled effect size after it was removed from the analysis, an individual study was doubted to have excessive influence. 


\section{CONCLUSIONS}

In summary, miR-20b, 21, 106b, 125a, 137, 141, 145, 146a, 196a, 196b, 206, 214, 218, 451, 486-5p and 506 demonstrate significantly prognostic value. Among them, miR-20b, 125a, 137, 141, 146a, 196a, 206, 218, $486-5 p$ and 506 are strong biomarkers of prognosis in GC.

\section{Author contributions}

Study concept and design: Yue Zhang and Yue-Hua Jiang.

Acquisition of data: Yue Zhang and Dong-Hui Guan.

Analysis and interpretation of data: Yue Zhang,

Dong-Hui Guan, Rong-Xiu Bi and Jin Xie.

Drafting of the manuscript: Yue Zhang.

Revision of manuscript: Yue Zhang, Dong-Hui Guan, Rong-Xiu Bi, Jin Xie, Chuan-Hua Yang and YueHua Jiang.

Supervision of work: Rong-Xiu Bi, Jin Xie, ChuanHua Yang and Yue-Hua Jiang.

All authors read and approved the final manuscript.

\section{CONFLICTS OF INTEREST} interest.

The authors declare that they have no conflicts of

\section{FUNDING}

This work was supported by the National Natural Science Foundation of China (No. 81673807).

Role of funding source: The funder had no role in study design, data collection and analysis, decision to publish, or preparation of the manuscript.

\section{REFERENCES}

1. Ueda T, Volinia S, Okumura H, Shimizu M, Taccioli C, Rossi S, Alder H, Liu CG, Oue N, Yasui W, Yoshida K, Sasaki H, Nomura S, et al. Relation between microRNA expression and progression and prognosis of gastric cancer: a microRNA expression analysis. Lancet Oncol. 2010; 11:136-146.

2. Wang YY, Ye ZY, Zhao ZS, Li L, Wang YX, Tao HQ, Wang HJ, He XJ. Clinicopathologic significance of miR$10 \mathrm{~b}$ expression in gastric carcinoma. Hum Pathol. 2013; 44:1278-1285.

3. Osawa S, Shimada Y, Sekine S, Okumura T, Nagata T, Fukuoka J, Tsukada K. MicroRNA profiling of gastric cancer patients from formalin-fixed paraffin-embedded samples. Oncol Lett. 2011; 2:613-619.

4. Ren C, Chen H, Han C, Fu D, Wang D, Shen M. High expression of miR-16 and miR-451 predicating better prognosis in patients with gastric cancer. J Cancer Res Clin Oncol. 2016; 142:2489-2496.

5. Wang M, Gu H, Wang S, Qian H, Zhu W, Zhang L, Zhao C, Tao Y, Xu W. Circulating miR-17-5p and miR-20a: Molecular markers for gastric cancer. Mol Med Rep. 2012; 5:1514-1520.

6. Komatsu S, Ichikawa D, Tsujiura M, Konishi H, Takeshita H, Nagata H, Kawaguchi T, Hirajima S, Arita T, Shiozaki A, Kubota T, Fujiwara H, Okamoto K, Otsuji E. Prognostic impact of circulating miR-21 in the plasma of patients with gastric carcinoma. Anticancer Res. 2013; 33:271-276.

7. Su ZX, Zhao J, Rong ZH, Wu YG, Geng WM, Qin CK. Diagnostic and prognostic value of circulating miR-18a in the plasma of patients with gastric cancer. Tumour Biol. 2014; 35:12119-12125.

8. Chen YJ, Wu H, Zhu JM, Li XD, Luo SW, Dong L, Liu TT, Shen XZ. MicroRNA-18a modulates P53 expression by targeting IRF2 in gastric cancer patients. J Gastroenterol Hepatol. 2016; 31:155-163.

9. Wu Q, Yang Z, An Y, Hu H, Yin J, Zhang P, Nie Y, Wu K, Shi Y, Fan D. MiR-19a/b modulate the metastasis of gastric cancer cells by targeting the tumour suppressor MXD1. Cell Death Dis. 2014; 5:e1144.

10. Wang H, Xiong M, Hu Y, Sun Y, Ma Q. MicroRNA-19b inhibits proliferation of gastric cancer cells by targeting B-cell CLL/lymphoma 3. Oncol Rep. 2016; 36:2079-2086.

11. Wu Q, Yang Z, Wang F, Hu S, Yang L, Shi Y, Fan D. MiR-19b/20a/92a regulates the self-renewal and proliferation of gastric cancer stem cells. J Cell Sci. 2013; 126:4220-4229.

12. Katada T, Ishiguro H, Kuwabara Y, Kimura M, Mitui A, Mori Y, Ogawa R, Harata K, Fujii Y. microRNA expression profile in undifferentiated gastric cancer. Int J Oncol. 2009; 34:537-542.

13. Xue TM, Tao LD, Zhang M, Xu GC, Zhang J, Zhang PJ. miR-20b overexpression is predictive of poor prognosis in gastric cancer. Onco Targets Ther. 2015; 8:1871-1876.

14. Park SK, Park YS, Ahn JY, Do EJ, Kim D, Kim JE, Jung K, Byeon JS, Ye BD, Yang DH, Park SH, Hwang SW, Jung HY, Myung SJ. MiR 21-5p as a predictor of recurrence in young gastric cancer patients. J Gastroenterol Hepatol. 2016; 31:1429-1435.

15. Jiang J, Zheng X, Xu X, Zhou Q, Yan H, Zhang X, Lu B, Wu C, Ju J. Prognostic significance of miR-181b and miR21 in gastric cancer patients treated with S-1/Oxaliplatin or Doxifluridine/Oxaliplatin. PLoS One. 2011; 6:e23271.

16. Xu Y, Sun J, Xu J, Li Q, Guo Y, Zhang Q. miR-21 is a promising novel biomarker for lymph node metastasis in patients with gastric cancer. Gastroenterol Res Pract. 2012; 2012:640168.

17. Hirata K, Suzuki H, Imaeda H, Matsuzaki J, Tsugawa H, Nagano O, Asakura K, Saya H, Hibi T. CD44 variant 9 expression in primary early gastric cancer as a predictive marker for recurrence. Br J Cancer. 2013; 109:379-386. 
18. Song J, Bai Z, Zhang J, Meng H, Cai J, Deng W, Bi J, Ma X, Zhang Z. Serum microRNA-21 levels are related to tumor size in gastric cancer patients but cannot predict prognosis. Oncol Lett. 2013; 6:1733-1737.

19. Wang D, Fan Z, Liu F, Zuo J. Hsa-miR-21 and HsamiR-21 and Hsa-miR-29 in tissue as potential diagnostic and prognostic biomarkers for gastric cancer. Cell Physiol Biochem. 2015; 37:1454-1462.

20. Wang W, Li F, Zhang Y, Tu Y, Yang Q, Gao X. Reduced expression of miR-22 in gastric cancer is related to clinicopathologic characteristics or patient prognosis. Diagn Pathol. 2013; 8:102.

21. Zuo QF, Cao LY, Yu T, Gong L, Wang LN, Zhao YL, Xiao B, Zou QM. MicroRNA-22 inhibits tumor growth and metastasis in gastric cancer by directly targeting MMP14 and Snail. Cell Death Dis. 2015; 6:e2000.

22. An Y, Zhang Z, Shang Y, Jiang X, Dong J, Yu P, Nie Y3, Zhao Q. miR-23b-3p regulates the chemoresistance of gastric cancer cells by targeting ATG12 and HMGB2. Cell Death Dis. 2015; 6:e1766.

23. Zhuang $\mathrm{K}$, Han $\mathrm{K}$, Tang H, Yin X, Zhang J, Zhang X, Zhang L. Up-regulation of plasma miR-23b is associated with poor prognosis of gastric cancer. Med Sci Monit. 2016; 22:356-361.

24. Wu X, Liu Z, Long J, Ge C, Song S. Knockdown of miR24 suppresses the proliferation, migration and invasion of gastric cancer cells and predicts a poor prognosis in gastric cancer. Int J Clin Exp Med. 2017; 10:2911-2917.

25. Li BS, Zuo QF, Zhao YL, Xiao B, Zhuang Y, Mao XH, Wu C, Yang SM, Zeng H, Zou QM, Guo G. MicroRNA-25 promotes gastric cancer migration, invasion and proliferation by directly targeting transducer of ERBB2, 1 and correlates with poor survival. Oncogene. 2015; 34:2556-2565.

26. Gong J, Cui Z, Li L, Ma Q, Wang Q, Gao Y, Sun H. MicroRNA-25 promotes gastric cancer proliferation, invasion, and migration by directly targeting F-box and WD-40 Domain Protein 7, FBXW7. Tumour Biol. 2015; 36:7831-7840.

27. Deng M, Tang HL, Lu XH, Liu MY, Lu XM, Gu YX, Liu JF, He ZM. miR-26a suppresses tumor growth and metastasis by targeting FGF9 in gastric cancer. PLoS One. 2013; 8:e72662.

28. Ding K, Wu Z, Wang N, Wang X, Wang Y, Qian P, Meng G, Tan S. MiR-26a performs converse roles in proliferation and metastasis of different gastric cancer cells via regulating of PTEN expression. Pathol Res Pract. 2017; S0344-0338:30366-30361.

29. Tsai MM, Huang HW, Wang CS, Lee KF, Tsai CY, Lu $\mathrm{PH}$, Chi HC, Lin YH, Kuo LM, Lin KH. MicroRNA26b inhibits tumor metastasis by targeting the KPNA2/cjun pathway in human gastric cancer. Oncotarget. 2016; 7:39511-39526. doi: 10.18632/oncotarget.8629.
30. Huang D, Wang H, Liu R, Li H, Ge S, Bai M, Deng T, Yao G, Ba Y. miRNA27a is a biomarker for predicting chemosensitivity and prognosis in metastatic or recurrent gastric cancer. J Cell Biochem. 2014; 115:549-556.

31. Park JL, Kim M, Song KS, Kim SY, Kim YS. Cell-free miR-27a, a potential diagnostic and prognostic biomarker for gastric cancer. Genomics Inform. 2015; 13:70-75.

32. Liu HT, Xing AY, Chen X, Ma RR, Wang YW, Shi DB, Zhang H, Li P, Chen HF, Li YH, Gao P. MicroRNA-27b, microRNA-101 and microRNA-128 inhibit angiogenesis by down-regulating vascular endothelial growth factor $\mathrm{C}$ expression in gastric cancers. Oncotarget. 2015; 6:3745837470. doi: 10.18632/oncotarget.6059.

33. Shang Y, Feng B, Zhou L, Ren G, Zhang Z, Fan X, Sun Y, Luo G, Liang J, Wu K, Nie Y, Fan D. The miR27b-CCNG1P53-miR-508-5p axis regulates multidrug resistance of gastric cancer. Oncotarget. 2016; 7:538-549. doi: 10.18632/ oncotarget.6374.

34. Liu HT, Wang YW, Xing AY, Shi DB, Zhang H, Guo XY, $\mathrm{Xu}$ J, Gao P. Prognostic value of microRNA signature in patients with gastric cancers. Sci Rep. 2017; 7:42806.

35. He B, Xiao YF, Tang B, Wu YY, Hu CJ, Xie R, Yang X, Yu ST, Dong H, Zhao XY, Li JL, Yang SM. hTERT mediates gastric cancer metastasis partially through the indirect targeting of ITGB1 by microRNA-29a. Sci Rep. 2016; $6: 21955$

36. Wang Y, Liu C, Luo M, Zhang Z, Gong J, Li J, You L, Dong L, Su R, Lin H, Ma Y, Wang F, Wang Y, et al. Chemotherapy-induced miRNA-29c/catenin- $\delta$ signaling suppresses metastasis in gastric cancer. Cancer Res. 2015; 75:1332-1344.

37. Wang H, Zhang X, Liu Y, Ni Z, Lin Y, Duan Z, Shi Y, Wang G, Li F. Downregulated miR-31 level associates with poor prognosis of gastric cancer and its restoration suppresses tumor cell malignant phenotypes by inhibiting E2F2. Oncotarget. 2016; 7:36577-36589. doi: 10.18632/ oncotarget.9288.

38. Hui WT, Ma XB, Zan Y, Wang XJ, Dong L. Prognostic significance of MiR-34a expression in patients with gastric cancer after radical gastrectomy. Chin Med J (Engl). 2015; 128:2632-2637.

39. Wei B, Huang QY, Huang SR, Mai W, Zhong XG. MicroRNA-34a attenuates the proliferation, invasion and metastasis of gastric cancer cells via downregulation of MET. Mol Med Rep. 2015; 12:5255-5261.

40. Zhang H, Li S, Yang J, Liu S, Gong X, Yu X. The prognostic value of miR-34a expression in completely resected gastric cancer: tumor recurrence and overall survival. Int J Clin Exp Med. 2015; 8:2635-2641.

41. Yang B, Huang J, Liu H, Guo W, Li G. miR-335 directly, while miR-34a indirectly modulate survivin expression and regulate growth, apoptosis, and invasion of gastric cancer cells. Tumour Biol. 2016; 37:1771-1779. 
42. Ren C, Wang W, Han C, Chen H, Fu D, Luo Y, Yao H, Wang D, Ma L, Zhou L, Han D, Shen M. Expression and prognostic value of miR-92a in patients with gastric cancer. Tumour Biol. 2016; 37:9483-9491.

43. Chen L, Jiang M, Yuan W, Tang H. Prognostic value of miR93 overexpression in resectable gastric adenocarcinomas. Acta Gastroenterol Belg. 2012; 75:22-27.

44. Yu BQ, Su LP, Li JF, Cai Q, Yan M, Chen XH, Yu YY, Gu QL, Zhu ZG, Liu BY. microRNA expression signature of gastric cancer cells relative to normal gastric mucosa. Mol Med Rep. 2012; 6:821-826.

45. Yang TS, Yang XH, Chen X, Wang XD, Hua J, Zhou DL, Zhou B, Song ZS. MicroRNA-106b in cancer-associated fibroblasts from gastric cancer promotes cell migration and invasion by targeting PTEN. FEBS Lett. 2014; 588:2162-2169.

46. Li X, Zhang Y, Shi Y, Dong G, Liang J, Han Y, Wang X, Zhao Q, Ding J, Wu K, Fan D. MicroRNA-107, an oncogene microRNA that regulates tumour invasion and metastasis by targeting DICER1 in gastric cancer. J Cell Mol Med. 2011; 15:1887-1895.

47. Inoue $T$, Iinuma $H$, Ogawa $E$, Inaba $T$, Fukushima $R$. Clinicopathological and prognostic significance of microRNA-107 and its relationship to DICER1 mRNA expression in gastric cancer. Oncol Rep. 2012; 27:1759-1764.

48. Chen Q, Ge X, Zhang Y, Xia H, Yuan D, Tang Q, Chen L, Pang X, Leng W, Bi F. Plasma miR-122 and miR192 as potential novel biomarkers for the early detection of distant metastasis of gastric cancer. Oncol Rep. 2014; 31:1863-1870.

49. Hashiguchi Y, Nishida N, Mimori K, Sudo T, Tanaka F, Shibata K, Ishii H, Mochizuki H, Hase K, Doki Y, Mori M. Down-regulation of miR-125a-3p in human gastric cancer and its clinicopathological significance. Int J Oncol. 2012; 40:1477-1482.

50. Nishida N, Mimori K, Fabbri M, Yokobori T, Sudo T, Tanaka F, Shibata K, Ishii H, Doki Y, Mori M. MicroRNA$125 a-5 p$ is an independent prognostic factor in gastric cancer and inhibits the proliferation of human gastric cancer cells in combination with trastuzumab. Clin Cancer Res. 2011; 17:2725-2733.

51. Dai J, Wang J, Yang L, Xiao Y, Ruan Q. MIR-125a regulates angiogenesis of gastric cancer by targeting vascular endothelial growth factor A. Int J Oncol. 2015; 47:1801-1810.

52. Wu JG, Wang JJ, Jiang X, Lan JP, He XJ, Wang HJ, Ma YY, Xia YJ, Ru GQ, Ma J, Zhao ZS, Zhou R. MiR$125 \mathrm{~b}$ promotes cell migration and invasion by targeting PPP1CA-Rb signal pathways in gastric cancer, resulting in a poor prognosis. Gastric Cancer. 2015; 18:729-739.

53. Wu S, Liu F, Xie L, Peng Y, Lv X, Zhu Y, Zhang Z, He $\mathrm{X}$. miR-125b suppresses proliferation and invasion by targeting MCL1 in gastric cancer. Biomed Res Int. 2015; 2015:365273.
54. Yang Z, Wang R, Zhang T, Dong X. MicroRNA-126 regulates migration and invasion of gastric cancer by targeting CADM1. Int J Clin Exp Pathol. 2015; 8:8869-8880.

55. Yue S, Shi H, Han J, Zhang T, Zhu W, Zhang D. Prognostic value of microRNA-126 and CRK expression in gastric cancer. Onco Targets Ther. 2016; 9:6127-6135.

56. Li CY, Liang GY, Yao WZ, Sui J, Shen X, Zhang YQ, Peng H, Hong WW, Ye YC, Zhang ZY, Zhang WH, Yin $\mathrm{LH}, \mathrm{Pu}$ YP. Identification and functional characterization of microRNAs reveal a potential role in gastric cancer progression. Clin Transl Oncol. 2017; 19:162-172.

57. Jiang H, Yu WW, Wang LL, Peng Y. miR-130a acts as a potential diagnostic biomarker and promotes gastric cancer migration, invasion and proliferation by targeting RUNX3 . Oncol Rep. 2015; 34:1153-1161.

58. Liu X, Yu H, Cai H, Wang Y. The expression and clinical significance of miR-132 in gastric cancer patients. Diagn Pathol. 2014; 9:57.

59. Cheng Z, Liu F, Wang G, Li Y, Zhang H, Li F. miR-133 is a key negative regulator of CDC42-PAK pathway in gastric cancer. Cell Signal. 2014; 26:2667-2673.

60. Zhang XT, Zhang Z, Xin YN, Ma XZ, Xuan SY. Impairment of growth of gastric carcinoma by miR-133-mediated Her-2 inhibition. Tumour Biol. 2015; 36:8925-8930.

61. Yan LH, Chen ZN, Li-Li, Chen J, Wei WE, Mo XW, Qin YZ, Lin Y, Chen JS. miR-135a promotes gastric cancer progression and resistance to oxaliplatin. Oncotarget. 2016; 7:70699-70714. doi: 10.18632/oncotarget.12208.

62. Gu Q, Zhang J, Hu H, Tan YE, Shi S, Nian Y. Clinical significance of MiR-137 expression in patients with gastric cancer after radical gastrectomy. PLoS One. 2015; 10:e0142377.

63. Zheng X, Dong J, Gong T, Zhang Z, Wang Y, Li Y, Shang Y, Li K, Ren G, Feng B, Li J, Tian Q, Tang S, et al. MicroRNA library-based functional screening identified miR-137 as a suppresser of gastric cancer cell proliferation. J Cancer Res Clin Oncol. 2015; 141:785-795.

64. Du Y, Chen Y, Wang F, Gu L. miR-137 plays tumor suppressor roles in gastric cancer cell lines by targeting KLF12 and MYO1C. Tumour Biol. 2016; 37:13557-13569.

65. Lu YB, Hu JJ, Sun WJ, Duan XH, Chen X. Prognostic value of miR-141 downregulation in gastric cancer. Genet Mol Res. 2015; 14:17305-17311.

66. Zhou X, Wang Y, Shan B, Han J, Zhu H, Lv Y, Fan X, Sang M, Liu XD, Liu W. The downregulation of miR200c/141 promotes ZEB1/2 expression and gastric cancer progression. Med Oncol. 2015; 32:428.

67. Huang M, Wu L, Qin Y, Li Z, Luo S, Qin H, Yang Y, Chen J. Anti-proliferative role and prognostic implication of miR141 in gastric cancer. Am J Transl Res. 2016; 8:3549-3557.

68. Zhang X, Yan Z, Zhang J, Gong L, Li W, Cui J, Liu Y, Gao Z, Li J, Shen L, Lu Y. Combination of hsa-miR-375 and hsa-miR-142-5p as a predictor for recurrence risk in gastric 
cancer patients following surgical resection. Ann Oncol. 2011; 22:2257-2266.

69. Naito Y, Sakamoto N, Oue N, Yashiro M, Sentani K, Yanagihara K, Hirakawa K, Yasui W. MicroRNA-143 regulates collagen type III expression in stromal fibroblasts of scirrhous type gastric cancer. Cancer Sci. 2014; 105:228-235.

70. Li JH, Chen X, Yan XJ, Li H, Chen SL. microRNA-143 acts as a prognostic marker in gastric cancer and its role in cell proliferation and invasion. Int J Clin Exp Pathol. 2016; 9:9810-9820.

71. Akiyoshi S, Fukagawa T, Ueo H, Ishibashi M, Takahashi Y, Fabbri M, Sasako M, Maehara Y, Mimori K, Mori M. Clinical significance of miR-144-ZFX axis in disseminated tumour cells in bone marrow in gastric cancer cases. Br J Cancer. 2012; 107:1345-1353.

72. Zhang Y, Wen X, Hu XL, Cheng LZ, Yu JY, Wei ZB. Downregulation of miR-145-5p correlates with poor prognosis in gastric cancer. Eur Rev Med Pharmacol Sci. 2016; 20:3026-3030.

73. Naito Y, Yasuno K, Tagawa H, Sakamoto N, Oue N, Yashiro M, Sentani K, Goto K, Shinmei S, Oo HZ, Yanagihara K, Hirakawa K, Yasui W. MicroRNA-145 is a potential prognostic factor of scirrhous type gastric cancer. Oncol Rep. 2014; 32:1720-1726.

74. Kogo R, Mimori K, Tanaka F, Komune S, Mori M. Clinical significance of miR-146a in gastric cancer cases. Clin Cancer Res. 2011; 17:4277-4284.

75. Hou Z, Xie L, Yu L, Qian X, Liu B. MicroRNA-146a is down-regulated in gastric cancer and regulates cell proliferation and apoptosis. Med Oncol. 2012; 29:886-892.

76. Luo Z, Li X, Zhao Z, Yang X, Xiao S, Zhou Y. MicroRNA146a affects the chemotherapeutic sensitivity and prognosis of advanced gastric cancer through the regulation of LIN52. Oncol Lett. 2017; 13:1386-1392.

77. Yoon SO, Kim EK, Lee M, Jung WY, Lee H, Kang Y, Jang YJ, Hong SW, Choi SH, Yang WI. NOVA1 inhibition by miR-146b-5p in the remnant tissue microenvironment defines occult residual disease after gastric cancer removal. Oncotarget. 2016; 7:2475-2495. doi: 10.18632/ oncotarget.6542.

78. Sakamoto N, Naito Y, Oue N, Sentani K, Uraoka N, Zarni Oo H, Yanagihara K, Aoyagi K, Sasaki H, Yasui W. MicroRNA-148a is downregulated in gastric cancer, targets MMP7, and indicates tumor invasiveness and poor prognosis. Cancer Sci. 2014; 105:236-243.

79. Smid D, Kulda V, Srbecka K, Kubackova D, Dolezal J, Daum O, Kucera R, Topolcan O, Treska V, Skalicky T, Pesta M. Tissue microRNAs as predictive markers for gastric cancer patients undergoing palliative chemotherapy. Int J Oncol. 2016; 48:2693-2703.

80. Zhang Z, Sun J, Bai Z, Li H, He S, Chen R, Che X. MicroRNA-153 acts as a prognostic marker in gastric cancer and its role in cell migration and invasion. Onco Targets Ther. 2015; 8:357-364.

81. Mi Y, Zhang D, Jiang W, Weng J, Zhou C, Huang K, Tang H, Yu Y, Liu X, Cui W, Zhang M, Sun X, Zhou Z, et al. miR-181a-5p promotes the progression of gastric cancer via RASSF6-mediated MAPK signalling activation. Cancer Lett. 2017; 389:11-22.

82. Cui M, Yue L, Fu Y, Yu W, Hou X, Zhang X. Association of microRNA-181c expression with the progression and prognosis of human gastric carcinoma. Hepatogastroenterology. 2013; 60:961-964.

83. Cao LL, Xie JW, Lin Y, Zheng CH, Li P, Wang JB, Lin JX, Lu J, Chen QY, Huang CM. miR-183 inhibits invasion of gastric cancer by targeting Ezrin. Int J Clin Exp Pathol. 2014; 7:5582-5594.

84. Xu L, Li Y, Yan D, He J, Liu D. MicroRNA-183 inhibits gastric cancer proliferation and invasion via directly targeting Bmi-1. Oncol Lett. 2014; 8:2345-2351.

85. Tan Z, Jiang H, Wu Y, Xie L, Dai W, Tang H, Tang S. miR185 is an independent prognosis factor and suppresses tumor metastasis in gastric cancer. Mol Cell Biochem. 2014; 386:223-231.

86. Li C, Lu S, Shi Y. MicroRNA-187 promotes growth and metastasis of gastric cancer by inhibiting FOXA2. Oncol Rep. 2017; 37:1747-1755.

87. Xu YJ, Fan Y. MiR-215/192 participates in gastric cancer progression. Clin Transl Oncol. 2015; 17:34-40.

88. Mu YP, Tang S, Sun WJ, Gao WM, Wang M, Su XL. Association of miR-193b down-regulation and miR196a up-regulation with clinicopathological features and prognosis in gastric cancer. Asian Pac J Cancer Prev. 2014; 15:8893-8900.

89. Chen X, Wang Y, Zang W, Du Y, Li M, Zhao G. miR-194 targets RBX1 gene to modulate proliferation and migration of gastric cancer cells. Tumour Biol. 2015; 36:2393-2401.

90. Sun M, Liu XH, Li JH, Yang JS, Zhang EB, Yin DD, Liu ZL, Zhou J, Ding Y, Li SQ, Wang ZX, Cao XF, De W. MiR$196 \mathrm{a}$ is upregulated in gastric cancer and promotes cell proliferation by downregulating p27(kip1). Mol Cancer Ther. 2012; 11:842-852.

91. Tsai MM, Wang CS, Tsai CY, Chen CY, Chi HC, Tseng YH, Chung PJ, Lin YH, Chung IH, Chen CY, Lin KH. MicroRNA-196a/-196b promote cell metastasis via negative regulation of radixin in human gastric cancer. Cancer Lett. 2014; 351:222-231.

92. Tsai MM, Wang CS, Tsai CY, Huang CG, Lee KF, Huang HW, Lin YH, Chi HC, Kuo LM, Lu PH, Lin KH. Circulating microRNA-196a/b are novel biomarkers associated with metastatic gastric cancer. Eur J Cancer. 2016; 64:137-148.

93. Lim JY, Yoon SO, Seol SY, Hong SW, Kim JW, Choi SH, Lee JS, Cho JY. Overexpression of miR-196b and HOXA10 characterize a poor-prognosis gastric cancer subtype. World J Gastroenterol. 2013; 19:7078-7088. 
94. Cui Z, Zheng X, Kong D. Decreased miR-198 expression and its prognostic significance in human gastric cancer. World J Surg Oncol. 2016; 14:33.

95. Song G, Zeng H, Li J, Xiao L, He Y, Tang Y, Li Y. miR199a regulates the tumor suppressor mitogen-activated protein kinase kinase kinase 11 in gastric cancer. Biol Pharm Bull. 2010; 33:1822-1827.

96. Tang H, Deng M, Tang Y, Xie X, Guo J, Kong Y, Ye F, Su Q, Xie X. miR-200b and miR-200c as prognostic factors and mediators of gastric cancer cell progression. Clin Cancer Res. 2013; 19:5602-5612.

97. Kurashige J, Mima K, Sawada G, Takahashi Y, Eguchi H, Sugimachi K, Mori M, Yanagihara K, Yashiro M, Hirakawa K, Baba H, Mimori K. Epigenetic modulation and repression of $\mathrm{miR}-200 \mathrm{~b}$ by cancer-associated fibroblasts contribute to cancer invasion and peritoneal dissemination in gastric cancer. Carcinogenesis. 2015; 36:133-141.

98. Valladares-Ayerbes M, Reboredo M, Medina-Villaamil V, Iglesias-Díaz P, Lorenzo-Patiño MJ, Haz M, Santamarina I, Blanco M, Fernández-Tajes J, Quindós M, Carral A, Figueroa A, Antón-Aparicio LM, Calvo L. Circulating miR-200c as a diagnostic and prognostic biomarker for gastric cancer. J Transl Med. 2012; 10:186.

99. Zhang HP, Sun FB, Li SJ. Serum miR-200c expression level as a prognostic biomarker for gastric cancer. Genet Mol Res. 2015; 14:15913-15920.

100. Liang M, Shi B, Liu J, He L, Yi G, Zhou L, Yu G, Zhou $\mathrm{X}$. Downregulation of miR203 induces overexpression of PIK3CA and predicts poor prognosis of gastric cancer patients. Drug Des Devel Ther. 2015; 9:3607-3616.

101. Imaoka H, Toiyama Y, Okigami M, Yasuda H, Saigusa S, Ohi M, Tanaka K, Inoue Y, Mohri Y, Kusunoki M. Circulating microRNA-203 predicts metastases, early recurrence, and poor prognosis in human gastric cancer. Gastric Cancer. 2016; 19:744-753.

102. Chen X, Liu XS, Liu HY, Lu YY, Li Y. Reduced expression of serum miR-204 predicts poor prognosis of gastric cancer. Genet Mol Res. 2016; 15.

103. Sacconi A, Biagioni F, Canu V, Mori F, Di Benedetto A, Lorenzon L, Ercolani C, Di Agostino S, Cambria AM, Germoni S, Grasso G, Blandino R, Panebianco V, et al. MiR204 targets Bcl-2 expression and enhances responsiveness of gastric cancer. Cell Death Dis. 2012; 3:e423.

104. Yang Q, Zhang C, Huang B, Li H, Zhang R, Huang Y, Wang J. Downregulation of microRNA-206 is a potent prognostic marker for patients with gastric cancer. Eur J Gastroenterol Hepatol. 2013; 25:953-957.

105. Shi H, Han J, Yue S, Zhang T, Zhu W, Zhang D. Prognostic significance of combined microRNA-206 and CyclinD2 in gastric cancer patients after curative surgery: a retrospective cohort study. Biomed Pharmacother. 2015; 71:210-215.
106. Hou CG, Luo XY, Li G. Diagnostic and prognostic value of serum microRNA-206 in patients with gastric cancer. Cell Physiol Biochem. 2016; 39:1512-1520.

107. Zhang C, Tian W, Meng L, Qu L, Shou C. PRL-3 promotes gastric cancer migration and invasion through a NF- $\kappa \mathrm{B}-$ HIF-1 $\alpha$-miR-210 axis. J Mol Med (Berl). 2016; 94:401-415.

108. Ma G, Dai W, Sang A, Yang X, Li Q. Expression of microRNA-211 is a novel prognostic indicator of poor survival in human gastric cancer. Int J Clin Exp Med. 2016; 9:7223-7228.

109. Li D, Li Z, Xiong J, Gong B, Zhang G, Cao C, Jie Z, Liu Y, Cao Y, Yan Y, Xiong H, Qiu L, Yang M, et al. MicroRNA-212 functions as an epigenetic-silenced tumor suppressor involving in tumor metastasis and invasion of gastric cancer through down-regulating PXN expression. Am J Cancer Res. 2015; 5:2980-2997.

110. Yang TS, Yang XH, Wang XD, Wang YL, Zhou B, Song ZS. MiR-214 regulate gastric cancer cell proliferation, migration and invasion by targeting PTEN. Cancer Cell Int. $2013 ; 13: 68$

111. Wang YW, Shi DB, Chen $\mathrm{X}$, Gao C, Gao $\mathrm{P}$. Clinicopathological significance of microRNA-214 in gastric cancer and its effect on cell biological behaviour. PLoS One. 2014; 9:e91307.

112. Chen DL, Zhang DS, Lu YX, Chen LZ, Zeng ZL, He MM, Wang FH, Li YH, Zhang HZ, Pelicano H, Zhang W, Xu RH. microRNA-217 inhibits tumor progression and metastasis by downregulating EZH2 and predicts favorable prognosis in gastric cancer. Oncotarget. 2015; 6:10868-10879. doi: 10.18632/oncotarget.3451.

113. Liu H, Yang Z, Zhang J, Zhu X. MicroRNA-217 in plasma: a potential biomarker in gastric cancer. Int J Clin Exp Med. 2017; 10:3313-3320.

114. Tie J, Pan Y, Zhao L, Wu K, Liu J, Sun S, Guo X, Wang B, Gang Y, Zhang Y, Li Q, Qiao T, Zhao Q, et al. MiR218 inhibits invasion and metastasis of gastric cancer by targeting the Robol receptor. PLoS Genet. 2010; 6:e1000879.

115. Xin SY, Feng XS, Zhou LQ, Sun JJ, Gao XL, Yao GL. Reduced expression of circulating microRNA-218 in gastric cancer and correlation with tumor invasion and prognosis. World J Gastroenterol. 2014; 20:6906-6911.

116. Wang XX, Ge SJ, Wang XL, Jiang LX, Sheng MF, Ma JJ. miR-218 tissue expression level is associated with aggressive progression of gastric cancer. Genet Mol Res. $2016 ; 15$.

117. Liu K, Li G, Fan C, Diao Y, Wu B, Li J. Increased Expression of MicroRNA-221 in gastric cancer and its clinical significance. J Int Med Res. 2012; 40:467-474.

118. Kim BH, Hong SW, Kim A, Choi SH, Yoon SO. Prognostic implications for high expression of oncogenic microRNAs in advanced gastric carcinoma. J Surg Oncol. 2013; 107:505-510. 
119. Fu Z, Qian F, Yang X, Jiang H, Chen Y, Liu S. Circulating miR-222 in plasma and its potential diagnostic and prognostic value in gastric cancer. Med Oncol. 2014; 31:164.

120. Li X, Zhang Y, Zhang H, Liu X, Gong T, Li M, Sun L, Ji G, Shi Y, Han Z, Han S, Nie Y, Chen X, et al. miRNA223 promotes gastric cancer invasion and metastasis by targeting tumor suppressor EPB41L3. Mol Cancer Res. 2011; 9:824-833.

121. Ma L, Chen Y, Zhang B, Liu G. Increased microRNA-223 in Helicobacter pylori-associated gastric cancer contributed to cancer cell proliferation and migration. Biosci Biotechnol Biochem. 2014; 78:602-608.

122. Zhang Y, Li CF, Ma LJ, Ding M, Zhang B. MicroRNA-224 aggrevates tumor growth and progression by targeting mTOR in gastric cancer. Int J Oncol. 2016; 49:1068-1080.

123. Shen Z, Li C, Zhang K, Yu W, Xiao H, Li B, Liu T. The up-regulation of miR-300 in gastric cancer and its effects on cells malignancy. Int J Clin Exp Med. 2015; 8:6773-6783.

124. Xu XD, He XJ, Tao HQ, Zhang W, Wang YY, Ye ZY, Zhao ZS. Abnormal expression of miR-301a in gastric cancer associated with progression and poor prognosis. J Surg Oncol. 2013; 108:197-202.

125. Li Y, Gao Y, Xu Y, Ma H, Yang M. Down-regulation of miR-326 is associated with poor prognosis and promotes growth and metastasis by targeting FSCN1 in gastric cancer. Growth Factors. 2015; 33:267-274.

126. Xue HG, Yang AH, Sun XG, Lu YY, Tian ZB. Expression of microRNA-328 functions as a biomarker for recurrence of early gastric cancer (EGC) after endoscopic submucosal dissection (ESD) by modulating CD44. Med Sci Monit. 2016; 22:4779-4785.

127. Yan Z, Xiong Y, Xu W, Gao J, Cheng Y, Wang Z, Chen $\mathrm{F}$, Zheng G. Identification of hsa-miR-335 as a prognostic signature in gastric cancer. PLoS One. 2012; 7:e40037.

128. Zhang JK, Li YS, Zhang CD, Dai DQ. Up-regulation of CRKL by microRNA-335 methylation is associated with poor prognosis in gastric cancer. Cancer Cell Int. 2017; $17: 28$.

129. Zheng L, Jiao W, Mei H, Song H, Li D, Xiang X, Chen Y, Yang F, Li H, Huang K, Tong Q. miRNA-337-3p inhibits gastric cancer progression through repressing myeloid zinc finger 1-facilitated expression of matrix metalloproteinase 14. Oncotarget. 2016; 7:40314-40328. doi: 10.18632/ oncotarget.9739.

130. Yin G, Zhou H, Xue Y, Yao B, Zhao W. MicroRNA-340 promotes the tumor growth of human gastric cancer by inhibiting cyclin G2. Oncol Rep. 2016; 36:1111-1118.

131. Ma F, Song H, Guo B, Zhang Y, Zheng Y, Lin C, Wu Y, Guan G, Sha R, Zhou Q, Wang D, Zhou X, Li J, Qiu X. MiR-361-5p inhibits colorectal and gastric cancer growth and metastasis by targeting staphylococcal nuclease domain containing-1. Oncotarget. 2015; 6:17404-17416. doi: 10.18632/oncotarget.3744.

132. Zhang PF, Sheng LL, Wang G, Tian M, Zhu LY, Zhang R, Zhang J, Zhu JS. miR-363 promotes proliferation and chemo-resistance of human gastric cancer via targeting of FBW7 ubiquitin ligase expression. Oncotarget. 2016; 7:35284-35292. doi: 10.18632/oncotarget.9169.

133. Wen X, Wu JQ, Peng W, Feng JF, Tang JH. MicroRNA-377 predicts poor clinical outcome of gastric cancer and induces tumorigenesis by targeting multiple tumor-suppressor genes. Oncol Rep. 2015; 34:203-210.

134. Zheng BQ, Long ZW, Chen J, Wang CM, Chen Y, Zhang RM, Wang YN, Shi YQ. Decreased expression of miR-378 is associated with local invasion, lymph node metastasis and poor prognosis in gastric cancer. Int J Clin Exp Pathol. 2016; 9:3774-3780.

135. Cao Q, Liu F, Ji K, Liu N, He Y, Zhang W, Wang L. MicroRNA-381 inhibits the metastasis of gastric cancer by targeting TMEM16A expression. J Exp Clin Cancer Res. 2017; 36:29.

136. Liu H, Gao Y, Song D, Liu T, Feng Y. Correlation between microRNA-421 expression level and prognosis of gastric cancer. Int J Clin Exp Pathol. 2015; 8:15128-15132.

137. Ge X, Liu X, Lin F, Li P, Liu K, Geng R, Dai C, Lin Y, Tang W, Wu Z, Chang J, Lu J, Li J. MicroRNA-421 regulated by HIF-1a promotes metastasis, inhibits apoptosis, and induces cisplatin resistance by targeting E-cadherin and caspase-3 in gastric cancer. Oncotarget. 2016; 7:24466-24482. doi: 10.18632/oncotarget.8228.

138. Zhu P, Zhang J, Zhu J, Shi J, Zhu Q, Gao Y. MiR-429 induces gastric carcinoma cell apoptosis through Bcl-2. Cell Physiol Biochem. 2015; 37:1572-1580.

139. Hong X, Xu Y, Qiu X, Zhu Y, Feng X, Ding Z, Zhang S, Zhong L, Zhuang Y, Su C, Hong X, Cai J. MiR-448 promotes glycolytic metabolism of gastric cancer by downregulating KDM2B. Oncotarget. 2016; 7:2209222102. doi: 10.18632/oncotarget.8020.

140. Wu Z, Wang H, Fang S, Xu C. MiR-449c inhibits gastric carcinoma growth. Life Sci. 2015; 137:14-19.

141. Bandres E, Bitarte N, Arias F, Agorreta J, Fortes P, Agirre X, Zarate R, Diaz-Gonzalez JA, Ramirez N, Sola JJ, Jimenez P, Rodriguez J, Garcia-Foncillas J. microRNA-451 regulates macrophage migration inhibitory factor production and proliferation of gastrointestinal cancer cells. Clin Cancer Res. 2009; 15:2281-2290.

142. Brenner B, Hoshen MB, Purim O, David MB, Ashkenazi K, Marshak G, Kundel Y, Brenner R, Morgenstern S, Halpern M, Rosenfeld N, Chajut A, Niv Y, Kushnir M. MicroRNAs as a potential prognostic factor in gastric cancer. World $\mathrm{J}$ Gastroenterol. 2011; 17:3976-3985.

143. Su Z, Zhao J, Rong Z, Geng W, Wang Z. MiR-451, a potential prognostic biomarker and tumor suppressor for gastric cancer. Int J Clin Exp Pathol. 2015; 8:9154-9160. 
144. Gao H, Liu P, Yang Y, Gao F. Decreased miR-452 expression and its tumor suppressive function in human gastric cancer. Int J Clin Exp Med. 2016; 9:16078-16085.

145. Liu J, Zhang J, Li Y, Wang L, Sui B, Dai D. MiR-455-5p acts as a novel tumor suppressor in gastric cancer by downregulating RAS18. Gene. 2016; 592:308-315.

146. Jing LL, Mo XM. Reduced miR-485-5p expression predicts poor prognosis in patients with gastric cancer. Eur Rev Med Pharmacol Sci. 2016; 20:1516-1520.

147. Chen H, Ren C, Han C, Wang D, Chen Y, Fu D. Expression and prognostic value of miR-486-5p in patients with gastric adenocarcinoma. PLoS One. 2015; 10:e0119384.

148. Ren C, Chen H, Han C, Fu D, Zhou L, Jin G, Wang F, Wang D, Chen Y, Ma L, Zheng X, Han D. miR-486-5p expression pattern in esophageal squamous cell carcinoma, gastric cancer and its prognostic value. Oncotarget. 2016; 7:1584015853. doi: 10.18632/oncotarget. 7417.

149. Zhou W, Zhang C, Jiang H, Zhang Z, Xie L, He X. MiR493 suppresses the proliferation and invasion of gastric cancer cells by targeting RhoC. Iran J Basic Med Sci. 2015; 18:1027-1033.

150. He W, Li Y, Chen X, Lu L, Tang B, Wang Z, Pan Y, Cai S, He Y, Ke Z. miR-494 acts as an anti-oncogene in gastric carcinoma by targeting c-myc. J Gastroenterol Hepatol. 2014; 29:1427-1434.

151. Zhang L, Ding Y, Yuan Z, Liu J, Sun J, Lei F, Wu S, Li S, Zhang D. MicroRNA-500 sustains nuclear factor- $\kappa \mathrm{B}$ activation and induces gastric cancer cell proliferation and resistance to apoptosis. Oncotarget. 2015; 6:2483-2495. doi: 10.18632/oncotarget.2800.

152. Fan D, Ren B, Yang X, Liu J, Zhang Z. Upregulation of miR-501-5p activates the wnt/ $\beta$-catenin signaling pathway and enhances stem cell-like phenotype in gastric cancer. J Exp Clin Cancer Res. 2016; 35:177.

153. Wu D, Cao G, Huang Z, Jin K, Hu H, Yu J, Zeng Y. Decreased miR-503 expression in gastric cancer is inversely correlated with serum carcinoembryonic antigen and acts as a potential prognostic and diagnostic biomarker. Onco Targets Ther. 2016; 10:129-135.

154. Deng J, Lei W, Xiang X, Zhang L, Yu F, Chen J, Feng M, Xiong J. MicroRNA-506 inhibits gastric cancer proliferation and invasion by directly targeting Yap1. Tumour Biol. 2015; 36:6823-6831.

155. Li Z, Liu Z, Dong S, Zhang J, Tan J, Wang Y, Ge C, Li R, Xue Y, Li M, Wang W, Xiang X, Yang J, et al. miR506 inhibits epithelial-to-mesenchymal transition and angiogenesis in gastric cancer. Am J Pathol. 2015; 185:2412-2420.

156. Sakimura S, Sugimachi K, Kurashige J, Ueda M, Hirata H, Nambara S, Komatsu H, Saito T, Takano Y, Uchi R, Sakimura E, Shinden Y, Iguchi T, et al. The miR-506induced epithelial-mesenchymal transition is involved in poor prognosis for patients with gastric cancer. Ann Surg Oncol. 2015; 22:S1436-1443.
157. Shang Y, Zhang Z, Liu Z, Feng B, Ren G, Li K, Zhou L, Sun Y, Li M, Zhou J, An Y, Wu K, Nie Y, Fan D. miR-508-5p regulates multidrug resistance of gastric cancer by targeting ABCB1 and ZNRD1. Oncogene. 2014; 33:3267-3276.

158. Li YR, Wen LQ, Wang Y, Zhou TC, Ma N, Hou ZH, Jiang ZP. MicroRNA-520c enhances cell proliferation, migration, and invasion by suppressing IRF2 in gastric cancer. FEBS Open Bio. 2016; 6:1257-1266.

159. Li R, Yuan W, Mei W, Yang K, Chen Z. MicroRNA 520d$3 p$ inhibits gastric cancer cell proliferation, migration, and invasion by downregulating EphA2 expression. Mol Cell Biochem. 2014; 396:295-305.

160. Zheng L, Jiao W, Song H, Qu H, Li D, Mei H, Chen Y, Yang F, Li H, Huang K, Tong Q. miRNA-558 promotes gastric cancer progression through attenuating Smad4-mediated repression of heparanase expression. Cell Death Dis. 2016; 7:e2382.

161. Shen B, Yu S, Zhang Y, Yuan Y, Li X, Zhong J, Feng J. miR-590-5p regulates gastric cancer cell growth and chemosensitivity through RECK and the AKT/ERK pathway. Onco Targets Ther. 2016; 9:6009-6019.

162. Chu D, Zhao Z, Li Y, Li J, Zheng J, Wang W, Zhao Q, Ji G. Increased microRNA-630 expression in gastric cancer is associated with poor overall survival. PLoS One. 2014; 9:e90526.

163. Chen X, Chen R, Wu W, Huang Z. MicroRNA-873 inhibits proliferation and induces apoptosis by targeting CXCL1 in gastric cancer. Int J Clin Exp Pathol. 2016; 9:10011-10019.

164. Zhang JX, Xu Y, Gao Y, Chen C, Zheng ZS, Yun M, Weng HW, Xie D, Ye S. Decreased expression of miR-939 contributes to chemoresistance and metastasis of gastric cancer via dysregulation of SLC34A2 and Raf/MEK/ERK pathway. Mol Cancer. 2017; 16:18.

165. Liu X, Ge X, Zhang Z, Zhang X, Chang J, Wu Z, Tang W, Gan L, Sun M, Li J. MicroRNA-940 promotes tumor cell invasion and metastasis by downregulating ZNF24 in gastric cancer. Oncotarget. 2015; 6:25418-25428. doi: 10.18632/oncotarget.4456.

166. Chen L, Lü MH, Zhang D, Hao NB, Fan YH, Wu YY, Wang SM, Xie R, Fang DC, Zhang H, Hu CJ, Yang SM. miR1207-5p and miR-1266 suppress gastric cancer growth and invasion by targeting telomerase reverse transcriptase. Cell Death Dis. 2014; 5:e1034.

167. Zheng H, Zhang F, Lin X, Huang C, Zhang Y, Li Y, Lin J, Chen W, Lin X. MicroRNA-1225-5p inhibits proliferation and metastasis of gastric carcinoma through repressing insulin receptor substrate- 1 and activation of $\beta$-catenin signaling. Oncotarget. 2016; 7:4647-4663. doi: 10.18632/ oncotarget.6615.

168. Shen L, Shan YS, Hu HM, Price TJ, Sirohi B, Yeh KH, Yang YH, Sano T, Yang HK, Zhang X, Park SR, Fujii M, Kang YK, Chen LT. Management of gastric cancer in 
Asia: resource-stratified guidelines. Lancet Oncol. 2013; 14:e535-547.

169. Ferlay J, Shin HR, Bray F, Forman D, Mathers C, Parkin DM. Estimates of worldwide burden of cancer in 2008: GLOBOCAN 2008. Int J Cancer. 2010; 127:2893-2917.

170. Varadhachary G, Ajani JA. Gastric cancer. Clin Adv Hematol Oncol. 2005; 3:118-124.

171. Wu WK, Lee CW, Cho CH, Fan D, Wu K, Yu J, Sung JJ. MicroRNA dysregulation in gastric cancer: a new player enters the game. Oncogene. 2010; 29:5761-5771.

172. Bartel DP. MicroRNAs: genomics, biogenesis, mechanism, and function. Cell. 2004; 116:281-297.

173. Krol J, Loedige I, Filipowicz W. The widespread regulation ofmicroRNA biogenesis, function and decay. Nat Rev Genet. 2010; 11:597-610.

174. Farooqi AA, Rehman ZU, Muntane J. Antisense therapeutics in oncology: current status. Onco Targets Ther. 2014; 7:2035-2042.

175. Song H, Sun W, Ye G, Ding X, Liu Z, Zhang S, Xia T, Xiao $\mathrm{B}, \mathrm{Xi}$ Y, Guo J. Long non-coding RNA expression profile in human gastric cancer and its clinical significances. J Transl Med. 2013; 11:225.

176. Li H, Yu B, Li J, Su L, Yan M, Zhu Z, Liu B. Overexpression of lncRNA H19 enhances carcinogenesis and metastasis of gastric cancer. Oncotarget. 2014; 5:2318-2329. doi: 10.18632/oncotarget.1913.

177. Zhang EB, Kong R, Yin DD, You LH, Sun M, Han L, $\mathrm{Xu}$ TP, Xia R, Yang JS, De W, Chen JF. Long noncoding RNA ANRIL indicates a poor prognosis of gastric cancer and promotes tumor growth by epigenetically silencing of miR-99a/miR-449a. Oncotarget. 2014; 5:2276-2292. doi: 10.18632/oncotarget.1902.

178. Yang F, Xue X, Zheng L, Bi J, Zhou Y, Zhi K, Gu Y, Fang G. Long non-coding RNA GHET1 promotes gastric carcinoma cell proliferation by increasing c-Myc mRNA stability. FEBS J. 2014; 281:802-813.

179. Endo H, Shiroki T, Nakagawa T, Yokoyama M, Tamai K, Yamanami H, Fujiya T, Sato I, Yamaguchi K, Tanaka N, Iijima K, Shimosegawa T, Sugamura K, Satoh K. Enhanced expression of long non-coding RNA HOTAIR is associated with the development of gastric cancer. PLoS One. 2013; 8:e77070.

180. Sun M, Jin FY, Xia R, Kong R, Li JH, Xu TP, Liu YW, Zhang EB, Liu XH, De W. Decreased expression of long noncoding RNA GAS5 indicates a poor prognosis and promotes cell proliferation in gastric cancer. BMC Cancer. 2014; 14:319.

181. Zhou B, Jing XY, Wu JQ, Xi HF, Lu GJ. Down-regulation of long non-coding RNA LET is associated with poor prognosis in gastric cancer. Int J Clin Exp Pathol. 2014; 7:8893-8898.

182. Hu Y, Wang J, Qian J, Kong X, Tang J, Wang Y, Chen H, Hong J, Zou W, Chen Y, Xu J, Fang JY. Long noncoding RNA GAPLINC regulates CD44-dependent cell invasiveness and associates with poor prognosis of gastric cancer. Cancer Res. 2014; 74:6890-6902.

183. Xu TP, Huang MD, Xia R, Liu XX, Sun M, Yin L, Chen WM, Han L, Zhang EB, Kong R, De W, Shu YQ. Decreased expression of the long non-coding RNA FENDRR is associated with poor prognosis in gastric cancer and FENDRR regulates gastric cancer cell metastasis by affecting fibronectin1 expression. J Hematol Oncol. 2014; 7:63.

184. Tierney JF, Stewart LA, Ghersi D, Burdett S, Sydes MR. Practical methods for incorporating summary time-to-event data into meta-analysis. Trials. 2007; 8:16. 